\title{
Manipulating Models and Grasping the Ideas They Represent
}

\author{
T. G. K. Bryce ${ }^{1}$ E. J. Blown ${ }^{1}$
}

Published online: 2 February 2016

(C) The Author(s) 2016. This article is published with open access at Springerlink.com

\begin{abstract}
This article notes the convergence of recent thinking in neuroscience and grounded cognition regarding the way we understand mental representation and recollection: ideas are dynamic and multi-modal, actively created at the point of recall. Also, neurophysiologically, re-entrant signalling among cortical circuits allows non-conscious processing to support our deliberative thoughts and actions. The qualitative research we describe examines the exchanges occurring during semi-structured interviews with 360 children age 3-13, including 294 from New Zealand (158 boys, 136 girls) and 66 from China (34 boys, 32 girls) concerning their understanding of the shape and motion of the Earth, Sun and Moon (ESM). We look closely at the relationships between what is revealed as children manipulate their own play-dough models and their apparent understandings of ESM concepts. In particular, we focus on the switching taking place between what is said, what is drawn and what is modelled. The evidence is supportive of Edelman's view that memory is non-representational and that concepts are the outcome of perceptual mappings, a view which is also in accord with Barsalou's notion that concepts are simulators or skills which operate consistently across several modalities. Quantitative data indicate that the dynamic structure of memory/concept creation is similar in both genders and common to the cultures/ethnicities compared (New Zealand European and Māori; Chinese Han) and that repeated interviews in this longitudinal research lead to more advanced modelling skills and/or more advanced shape and motion concepts, the results supporting hypotheses (Kolmogorov-Smirnov alpha levels .05; $r_{s}: p<.001$ ).
\end{abstract}

Keywords Memory - Mental models - Conceptual coherence · Framework theory · Modal skills and simulations $\cdot$ Re-entrant signalling

You cannot depend on your eyes when your imagination is out of focus. Mark Twain (1989)

\footnotetext{
T. G. K. Bryce

t.g.k.bryce@strath.ac.uk

1 University of Strathclyde, Glasgow, UK
} 


\section{Introduction}

Young people's understandings of the visible cosmos (the Sun, the Moon, planet Earth and the stars) have been the subject of research for many decades, and much is known about the acquisition - the relatively slow acquisition — of accurate, scientific ideas. Following in the Piagetian tradition (see Piaget 1929, 1930), the major focus for researchers has been on what young people say in response to questions, on their verbal explanations during semistructured interviews for events such as daytime and night-time, sunrise and sunset, the seasons, lunar phases, and eclipses. Secondly, but to a lesser extent, influenced by the pioneering work of Nussbaum and Novak (1976), Nussbaum (1979) and the later work of Klein (1982), Sneider and Pulos (1983) and Vosniadou and Brewer (1992, 1994), children's drawings of the Earth, Sun and Moon (ESM) have provided an alternative medium of interpretation of children's cosmological concepts. Thirdly, models-created by children from either clay (see Brewer et al. 1987) or play-dough (see Bryce and Blown 2006), or the use of cultural artefacts like plastic or styrofoam models of globes by teachers or researchers (see Schoultz et al. 2001; Vosniadou et al. 2005) — have also been interpreted for insights into what they reveal, often in complementary but not always consistent ways.

Interviews with young people endeavour to tap into their previous learning, of course, and conversational exchanges pivot on what subjects can recollect or bring to mind at that point. It is readily apparent that, where circumstances permit-in research, in everyday teaching and in ordinary conversations-people sometimes naturally shift from verbal exchange to sketching their thoughts or, more rarely (particularly in the case of 3D objects and concepts), devising a model to show what they mean or perhaps signalling an indication with their hands and arms. (Classical examples in the history of science with regard to models include the Rutherford-Bohr model of the atom and the Watson-Crick model of DNA.) Relatively few researchers have explored the differences stemming from these modalities and the variations in apparent knowledge when subjects change from explanation to drawing to modelling. The research described here looked closely at these variations and the ways in which subjects revealed comprehension when they changed modes in the course of a semi-structured interview. That is, we focused on the switching taking place between what was said, what was drawn and what was modelled.

Important, however, is the underpinning theorising involved in all of these considerations. In the aforementioned literature on children's cosmologies, educational researchers and developmental psychologists have conventionally reasoned, though not always explicitly in the case of ESM investigations, that the recollections from memory which children are deploying as they answer questions and problem-solve are in the form of mental models. That is, in common with mainstream cognitive psychologists, it is usually presumed that subjects use some kind of internal representation of the world, as they consider things (see Gentner and Stevens 1983; Johnson-Laird 1983), whether these representations are silent verbalisations or images of some kind (in 2 or 3 dimensions). In our own previous investigations exploring age effects, gender and cultural differences in conceptual development (Bryce and Blown 2006), we implicitly assumed children's cosmological ideas to reflect the use of mental models. These had much in common with Nussbaum and Novak's (1976) Earth notions and appeared to be based on a representative form of memory. Although they recognised that their Earth notions were "not the only ones prevalent in the wide population of children" (p. 542) and Vosniadou (1992) described mental models as "synthetic and dynamic structures" (p. 348), both Earth notions and mental models have tended to be interpreted as static forms used to categorise children's concepts. Such a view 
has been reinforced by diagrams of the various models being commonly used as illustrations of same. Nevertheless, research has shown that variations in methodology and interview technique can influence the range of mental models elicited (see Frède et al. 2011; Nobes et al. 2003; Panagiotaki et al. 2006; Schoultz et al. 2001). This suggests that children's cosmologies are richer than the categorisation systems used to classify them.

Most importantly, recent experimentation and theoretical deliberations in other branches of science provide a quite different alternative and, in order to contextualise the present research, we need to appreciate what neurophysiologists and neuropsychologists now say about the multi-modality of recollection: mental representation is now understood very differently from what pioneering cognitive scientists envisaged. Our rationale for the research and the analysis described in this article was framed by these new considerations. Before we examine them, it is pertinent to comment on the terms mental models and modelling more generally.

\section{Mental Models and Modelling in the Science Education Literature}

A review of relevant literature reveals that educational researchers have, for some time, explored students' construction and use of models as part of their instruction in science, often relating issues to the role of models and thought experiments in the discipline itself. With the Nature of Science (NoS) figuring in recent science curricula, some researchers have explored the relationships between what students experience in the classroom and laboratory, how well they grasp related scientific content as specified in school curricula, and how scientists construe their own speciality-see, for example, Matthews' (2007) introductory chapter in the special issue of Science \& Education 16(7) and the articles therein. Adúriz-Bravo's (2013) philosophical analysis makes the case for a "semantic" view of scientific models for science education to be pursued. Thought experiments, both in the conduct of science and productively in relation to its teaching, have been considered in recent articles by Reiner and Burko (2003), Galili (2009), Blown and Bryce (2012) and Kösem and Özdemir (2014). In the article by Justi and Gilbert (2002) and the text by Gilbert and Boulter (2012), both models and modelling are discussed extensively.

The scientific activity of modelling seeks to make real-world phenomena easier to deal with, essentially by simplifying matters and allowing exploration and testing to be carried out on factors of interest and relevance. Proponents of "model-based" learning in education consider that students can gain insights into the scientific ideas involved through some form of activity. Where this is used in an iterative fashion, the teacher's role becomes more akin to that of a facilitator, managing how the students go about weighing up ideas, laboratory results and previous learning. Grandy (2003) considers whether "model-based" is akin to "theorising" and looks at the relationship between implicit mental models and explicit external models. He argues that more attention should be paid to the mathematical nature of many models in science. Halloun (2006) takes the argument further, contending that education in science should be more aligned with how scientists use modelling in their work and therefore that pedagogy in science education necessarily should be concerned with model-based learning. He reasons that teacher mediation of appropriate kinds can improve student learning, reduce attrition rates and narrow gaps in achievement between students from different backgrounds.

Examples of research looking at students' mental models drawing on the historical perspective include the report by Spiliotopoulou-Papantoniou (2006) on pupils' drawings 
of "how they imagine the universe to be", comparing them with past and present scientific conceptions. While she found no overall analogical evolution of the ideas between these two fields, some children's models resembled historical cases. A second example is the study by Feigenberg et al. (2002) which dealt with the problem of relating the distance and scale of the cosmic bodies in the solar system, comparing historic as well as contemporary views of scientists with children's intuitive understandings and their grasp of the relative dimensions involved. The authors argue that pupil activities be broadened to include laboratory work based on historical experiments. Thirdly, Thijs and Van Den Berg's (1995) report examined children's alternative frameworks cross-culturally, finding some of them to be universal and others to be culture specific. The researchers conclude that the effectiveness of ways of remediating alternative conceptions is strongly influenced by cultural aspects of the teaching-learning process. Fourthly, with regard to motion and mechanics (considered by many writers to be fundamental to basic science), Ebison (1993) argues that the features of Aristotelian physics (eventually reformulated by Galileo and Newton) were rooted in common sense, thus explaining why young persons' understandings are so resistant to change. Fifthly, and again from a historical perspective, Lauginie (2013) examines how scientists eventually understood that light had a velocity and argues that key points in that history ought to be brought into science discussions. Sixthly, and concerned explicitly with the pre-service preparation of primary teachers to teach science, de Hosson and Décamp (2014) describe the use of ancient Chinese and Greek astronomical data as part of a training sequence in elementary astronomy. The researchers relate their justification for the success of their materials to the central arguments involved in teaching the Nature of Science.

\section{Neuroscience and Recent Ideas in Cognition}

Recent advances in neuroscience have revealed the complex morphology of the human brain, in particular the immense richness and intricacy of its neural interconnections and what these achieve. The new developments have transformed what is understood to be happening during what we think of as recognition or recall, moving away from the conventional notion of the extraction of items from a fixed storage or database to the idea of memory as skill rather than content. This radical view considers memory to be a dynamical system whereby recall consists of the skill or ability to create and recreate concepts by way of imagery and simulations, and to translate these into representations by way of language, drawings, models and other modalities. Edelman $(2005,2006)$ has emphasised the huge redundant capacity involved in neurons and neural networks and how the networks function to create working memory and recollection (and indeed consciousness itself). He argues, with support from modern scanning technologies and investigations using noninvasive magneto-encephalography, that groups of neurons undergo selection during human maturation, learning and recall. These changes continue to be developed and modified as we interact with the environment. In emphasising the plasticity of the brain throughout an individual's life, Edelman states:

Not only is the fine structure of each brain unique, but the principles of Neural Darwinism [selection at the synaptic level] lead directly to the notion of degeneracy: different brain structures can carry out the same function or lead to the same output (Edelman 2006, p. 57).

The neural group selection that Edelman describes means that the resulting mental processing is anything but a reflection of repeated "programming". Crucially, what is 
called re-entrant signalling ${ }^{1}$ occurs so that there is an ongoing interchange of signals taking place in parallel between brain maps, these continuously relating one to another in time and space. "[T]he overall result is that reentry allows the integration, at many levels, of functionally segregated maps" (Edelman and Tononi 1996, p. 186, emphasis added). Or, in Edelman's more recent text, what happens is "the widespread synchronisation of the activity of widely distributed neuronal groups" (Edelman 2005, p. 41). This means that there is a steady interplay between numerous networks mediating the processes which result in what are traditionally understood as mental representation, imaging, conceptualisation, skilled manipulation and so forth-and the interplay is highly adaptive. For example, to quote Edelman again:

The mapped connections from the sense of touch in the hand through the thalamus to the region of somatosensory cortex are variable and plastic, even in adults. The subregions in the somatosensory cortex mapping the fingers dynamically shift all their boundaries as a result of excessive use of even one finger-a shift in the context of use (Edelman 2005, p. 36).

These ideas from neuroscience interestingly connect with the thinking of those cognitive theorists who have questioned the idea of concepts as representations and posit them not as models of reality such as mental models but rather as simulators or skills in creating such images consistently over a range of modal stimuli and responses (see Barsalou 2003). Van Gelder (1998) has even gone as far as to dispense with the idea of representation entirely in his consideration of the relationships between skill and knowledge. We have not resorted to this in our own previous work on the coherence of conceptions (see, for example, Blown and Bryce (2010) where we have assessed young people's understandings via different modalities - talking, drawing and play-dough modelling), but we have certainly demonstrated conceptual coherence in children's thinking compatible with both a representational connotation and a cognitive skill interpretation of concepts. We found no evidence in support of the counter-theory of knowledge-in-pieces proposed by diSessa $(1988,2008)$ and advocated by Nobes et al. (2003). The original arguments by Murphy and Medin (1985, pp. 289-290) that peoples' theories of the world embody conceptual knowledge have been reiterated in modern terms by Vosniadou et al. (2008, p. 4): "At the heart of our theoretical approach is the idea that initial explanations of the physical world in naïve physics are not fragmented observations but form a coherent whole, a framework theory". ${ }^{2}$ Thus, we have used the expression knowledge-skill compounding in the development of children's thought processes "whereby improved conceptual skill may be effected by a complex interaction of socio-cultural factors including cultural awareness, priority given to science in society, and emphasis on astronomy in the science curriculum" (Blown and Bryce 2010, p. 37).

Edelman is firmly dismissive of the common usages of representation and is a pains to say that brain morphology does not permit any equation between "meaning" and "mental representation" in the ways in which both of these terms are normally used. To quote him again:

There are no functional states that can be uniquely equated with defined or coded computational states in individual brains and no processes that can be equated with the execution of algorithms. Instead, there is an enormously rich set of selectional repertoires of neuronal groups whose

\footnotetext{
${ }^{1}$ Some writers, like Rose, consider the term re-entry to be confusing and refer to the multiple and reciprocal interconnections among the module circuitry in the brain (see Rose 2006, 2007). Confusingly, different writers use several spellings (re-entry, re-entrant, reentry, reentrant) all meaning the same thing.

2 An update on this has been recently published by Vosniadou and Skopeliti (2014) and will be discussed in the concluding sections of the present article.
} 
degenerate responses can, by selection, accommodate the open-ended richness of environmental input, individual history, and individual variation (Edelman 2005, p. 111).

The point here is that the neuroscience and the research on grounded cognition (whereby "modal simulations, bodily states, and situated action underlie cognition" according to Barsalou 2008, p. 617) resolve any question about the primacy of any one mode of thinking, or of its dependent relationship upon another. Barsalou points to how neuroimaging research has confirmed the central role that simulation ${ }^{3}$ plays in conceptual processing. Citing Martin (2001, 2007), he states:

When conceptual knowledge about objects is represented, brain areas that represent their properties during perception and action become active. In particular, brain areas that represent the shape and color of objects (fusiform gyrus), the motion they exhibit (middle and superior temporal lobe), and the actions that agents perform on them (premotor and parietal areas) become active to represent these properties conceptually (Barsalou 2008, p. 627).

We should recognise that neuroscientists now urge us to view thinking as involving mutually supportive, self-correcting and simultaneously adjusting processes instigated by different modalities - all the time, naturally. The child who is explaining in words his/her understanding of the movement of the Earth around the Sun, then switches to his/her own drawing of these bodies to show what he/she means, or upon the request of an interviewer, models them with play-dough to represent them three-dimensionally, does so because his/ her brain's wiring enables such capabilities - either simultaneously or near simultaneously in time. And, by switching modes, whether naturally at his/her own instigation, or through directed questioning (Socratic dialogue), the child modifies his/her ideas (or at least endeavours to do so, often to clarify meaning).

To take a particular finding from our research on children's cosmologies, it is interesting that there is a strong correlation between children's ability to model Earth-Sun-Moon configurations as spherical bodies, and the ability to model the rotation and revolution of these bodies; i.e. modelling motion scientifically appears to be strongly related to modelling shape scientifically (if a child models the Earth, Sun and Moon as spheres, they are more likely to be able to model rotation and revolution scientifically). Similarly, there are strong correlations between children's verbal descriptions of cosmological concepts such as Earth Shape and Earth Motion, and children's drawings of Earth Shape and Earth Motion (see Results). There may be several factors at work here, such as how these ideas figure during instruction or in presentations which children come across in books and television, but one possibility relates to the now-demonstrated multi-modality of the sensory-motor system - see Gallese and Lakoff (2005) whose work we will return to in the final section. Modelling motion scientifically and modelling shape scientifically may be pertinent reflections of that multi-modality. Our first consideration was that the coherence among the shape and motion concepts from three media of expression might indicate that there is a deeper underlying structure [possibly founded on roundness, circularity and sphericity (see Fig. 1 in due course), and possibly in turn based on an unconscious non-

\footnotetext{
3 Cognitive theorists use the term simulation to mean the mental re-enactment of perceptual, motor and introspective states acquired during our previous experiences with the world, the actions of our own bodies, and our minds. According to Barsalou (2003): "A concept is not a single abstracted representation for a category, but is instead a skill for constructing idiosyncratic representations tailored to the current needs of situated action... a concept is a simulator that constructs an infinite set of specific simulations..." (p. 521). Thus, from a global dynamic perspective a concept is both a skill and a simulator, and one can imagine it as a skill in creating simulations. However, from a domain-specific perspective, representation of concepts requires cognitive skill to imagine, create and regenerate the concepts (such as Earth's shape or motion), and dexterous and motor skills to form the shape of the concept and model its rotation and revolution.
} 


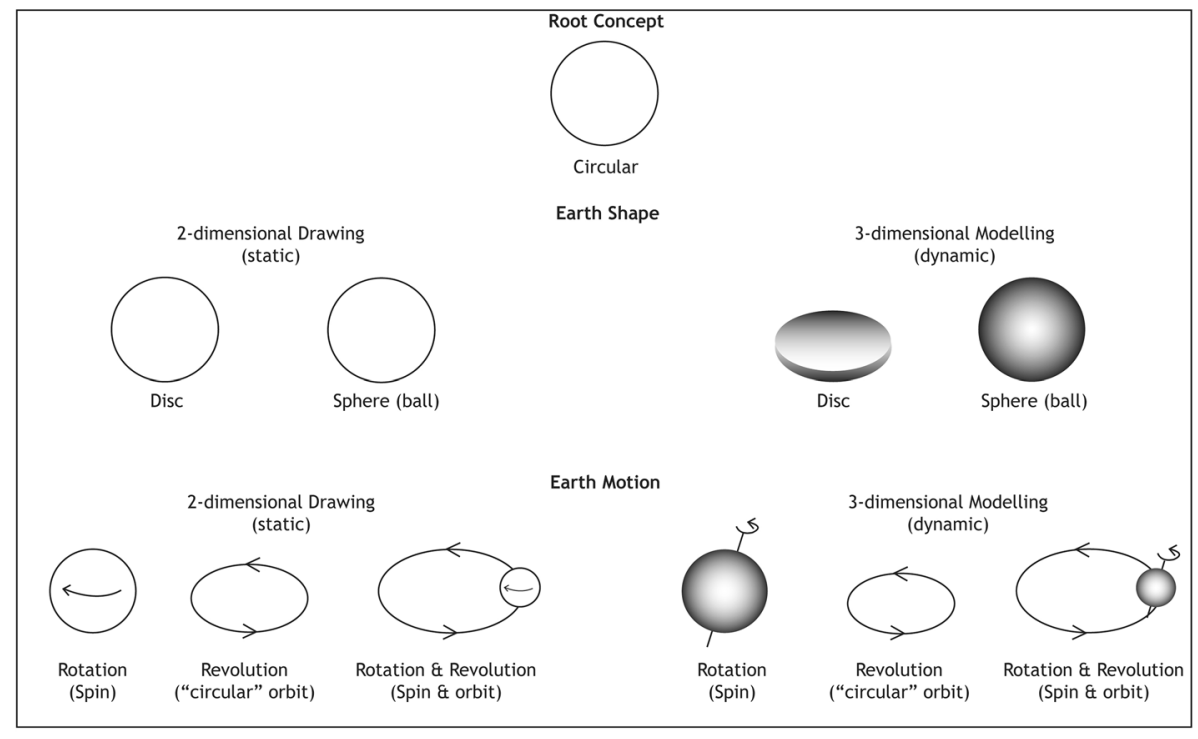

Fig. 1 Variations of the root concept of circularity in drawing and modelling Earth Shape and motion concepts

representative substrate as suggested by Edelman 2005, p. 104, see below] from which specific concepts of shape and motion draw static and kinetic images. But, regardless of whether or not this is the case, the coherence does support the notion that children's ideas are both dynamic and multi-modal.

That is, to generalise, such correlations detected in developmental research are consistent with the consequences of the functional properties of the re-entrant structures revealed by neuroscience research. We think multi-modally, adjusting our reasoning as we go along, exploiting metaphors and borrowed images as best we can. A picture that we draw, or a model that we make, neither precedes nor follows an explanation that we give in attempting to indicate what we mean; each modality we deploy reveals some of our meaning, and in turn our efforts may adjust our thinking - though the different ways in which we do so may be more or less helpful to those who attend to us.

The switching of ideas which takes place as we reason or problem-solve necessarily involves shifts between ideas which we are consciously addressing and ideas which, moments before, we were not aware of, but somehow "come to mind". Recent research is beginning to illuminate what is taking place. According to Dresp-Langley (2012) and referring mainly to neuro-psychological investigations, “... a large number of studies have shown that non-conscious brain processes influence perceptions and representations embedded in ongoing conscious experience" (p. 2). She argues, therefore, that far more is "known" than our deliberative, mindful and immediate thinking would suggest. And there is experimental evidence that the individual may be unaware of the support which nonconscious processing can give to conscious thoughts. So much so, that “... human decisions and actions based on so-called intuition are quite often timely and pertinent and reflect the astonishing ability of the brain to exploit non-conscious representations for conscious action, effortlessly and effectively" (Dresp-Langley 2012, p. 8). 
The exact mechanisms in the neuro-physiology remain unclear, but Edelman's reentrant signalling in cortical circuits is the main contender: bottom-up representations probably activate relevant structures but not sufficiently to trigger long-term memory signals. The latter requires the coincidence of activity among several neural circuits. With a critical amount of connectivity between neural structures, re-entrant signalling triggers conscious experience. Generalising beyond this, memory is therefore "... a dynamic, recategorical system property, not a fixed storage of all the variants of a scene, say of a familiar room..." (Edelman 2006, p. 59).

According to Rosenfield (1988), throughout the twentieth century an erroneous "fixed storage" view of memory persisted, influencing the work undertaken by researchers in several fields. It did so despite the prominent psychologists, e.g. James, Bartlett (Bartlett used the term constructive memory), Piaget and Bransford-all of whom had earlier stressed the activity involved in remembering (remembering is "re-membering") and that any present context shapes the way in which recollection operates. Piaget, for example, considered that "human knowledge is essentially active" and was "opposed to the view of knowledge as a copy, a passive copy of reality" (Piaget 1970, p. 15). Clancey's lengthy and detailed review of Rosenfield's text published in Artificial Intelligence (Clancey 1991) strove emphatically to disabuse researchers in that field of the notion that memory consisted of "addressable, localizable, retrievable structures (stored representations)" (p. 257). Perhaps a number of twenty-first century researchers in developmental and educational research need similar reminders about the nature of recollection processes when young people, or adults, reason during interviews designed to reveal how they grasp ideas and form an understanding of the world around them.

In respect of the present paper, we chose to analyse a set of interview data focusing specifically on inter-modal concept creation to provide a test of this recent thinking by neuroscientists and to challenge existing research in the field of children's cosmologies, research which, thus far, has under-theorised the nature of the conceptual knowledge which is tapped during interrogative dialogue. ESM studies continue to be a rich field for exploring preschool and in-school concept acquisitions and their inter-relationships. The investigation should provide a test of this neglected aspect of that developmental research through focusing on what remembering is now thought to entail.

\section{An Empirical Study}

\subsection{Background}

The investigation involved detailed one-to-one interviewing of young people regarding their grasp of the shapes and motions of the Earth, Sun and Moon. In the light of the literature reviewed above, we looked closely at the relationships between what was being conveyed by subjects via the aforementioned modes (verbal explanation, drawing and modelling), particularly where subjects switched between what they said, what they drew and how they created and manipulated their play-dough models. We sought to explore how the substance of what subjects were expressing altered and in what ways when they changed modes, and whether there were any patterns relating to the ages of the young people concerned. For example, given what is known developmentally about shifts from geocentric to heliocentric ideas about the Earth and the Sun, would there always be consistencies between a child's explanations, drawings and what they modelled, or might 
the act of modelling result in a change of mind (particularly within the dynamic context of Socratic dialogue during Piagetian interviews)? Or, with even younger children, how do changes from disc-shaped to spherical conceptions of the Earth manifest themselves? Because of their dynamic 3D nature, the models created by children would allow them, in association with pertinent questions from the researcher, to clarify their ideas of shape and motion. Verbal language acts as a universal medium and modality and as such is the foundation of interpretation (although problematic with young children age 3-5 and in ethnographic research). Drawing provides a 2D perspective, but it takes plastic modelling to enable critical comparison between modalities. Possible cognitive conflicts resulting from a child's failure to interconnect their thinking in different modes, and attempts to resolve them, should be manifest as switching of model shape or motion, and reflective of processes similar to Edelman's (2005) re-entrant signalling whereby information from verbal language, $2 \mathrm{D}$ drawings and 3D models are compared. The term cognitive conflict (or conceptual conflict) as used by developmental psychologists refers to instances where an individual may face inconsistencies between two (or more) of their thoughts. Piaget's notion of equilibration alludes to the natural tendency for the individual to try to reconcile these (see Piaget 1985). In Schnotz and Preuß's (1997) review of the literature on conceptual change, the authors state that: "conceptual change is a radical restructuring of an individual's intuitive theory-a sudden shift to a new perspective based on alternative core concepts and new relationships between them" (Schnotz and Preuß 1997, p. 187). Researchers (see, for example, Dreyfus et al. 1990) have debated whether or under what conditions conflicting thoughts are actually reconciled-with old conceptions being replaced by new (different/better) understandings, or whether both are maintained, i.e. that old and new conceptions coexist. Switching between such conceptions is therefore possible, indeed rather common. Thus, in the context of the present research, we sought to explore inter-modal as well as intra-modal switching, hoping to illuminate conceptual changes detectable when subjects try to reconcile conflicting thoughts, and/or images, and/ or models. Referring to the neurological revelations of Edelman, it is likely that re-entrant signalling accounts for cognitive conflict and switching as part of a dynamic system.

An example in the present context is that a child may say that he/she believes that the Earth is "round" as a result of verbal information from teachers, parents and peers, but may not have thought too much about what this means until asked to draw the Earth. A 2D representation lacks depth in two senses: it lacks linguistic precision and it is "flat". Analogy by the interviewer asking: "Round like a ball or round like a disc (pancake)?" can help to clarify what is meant; the child is forced to question himself/herself on what "round" means, to resolve the ambiguity in "roundness". Modelling in three dimensions provides the child with the means to express the concept, limited only by their concept skill or ability to create and express (i.e. model) the concept $c$.f. Barsalou's multi-modal concept skill (Barsalou 2003) and Clancey (1999). Sometimes it is only when asked to compare the three outputs from different modalities that the child experiences a "Eureka moment" and, at best, realises that they have a coherent concept of the Earth and its "roundness".

\subsection{Research Questions}

Refining our thinking about what we sought to examine in this area of children's cosmologies, we considered four research questions (RQs) in the light of the key literature. Would there be evidence that: 
RQ1 (a) Does thinking involve mutually supportive, self-correcting and simultaneously adjusting processes instigated by different modalities - all the time, naturally, in accord with Edelman's re-entrant signalling?

RQ1 (b) Is "memory non-representational and are concepts the outcome of the brain's mapping its own perceptual maps leading to generalities or 'universals"” (Edelman 2005, p. 104)?

RQ2 (a) Does memory, consisting not in the storage and retrieval of symbols, result from a dynamical process of activation, whereby concepts reflect deep links between perceptual motor skills and higher-level cognition (c.f. Clancey 1999)?

RQ2 (b) Are concepts skills or simulators for constructing idiosyncratic representations (Barsalou 2003)?

Addressing these questions in the context of ESM should provide both a test of new neuroscientific thinking about remembering and allow the reconsideration of previous researches that have overlooked the inter-relationships between what subjects can describe, draw or model. An important rider to the research strategy is to recognise the differences between the semi-structured situations we set up and what might pertain with individuals in more natural settings where there are fewer cues and constraints (as may be more often the case in class teaching sessions or private study and learning). The interview data which follow certainly show that individuals vary in the extent of the switching - the inter-modal cross-referencing - they make and therefore in the integration of their thinking. The experimental strategy limits the generalisations we might make to the non-constrained/ non-facilitated circumstances that occur in everyday life. Thus, it has to be acknowledged that the research questions set out above, particularly RQ2 (b), are framed in that light. The testing of the research question is limited by the very nature of the strategy deployed.

The overall strategy and research questions required a close scrutiny of qualitative data in order to permit comparisons with the findings from previous researches which have overlooked inter-modal considerations. However, previous investigations (including our own) have often also looked at quantitative data in the search for possible effects of gender and country of origin (culture) and ethnicity, and whether repeated surveying, even years apart, would be influential (developmental effects are of course easily demonstrated). We therefore considered that a secondary analysis should also check these variables for the subjects involved in the circumstances of the new analysis. Potentially, female and male interviewees, Chinese and New Zealand subjects, and in the latter case NZ (European) and NZ (Māori) participants might react differently to the close scrutiny required in semi-structured interviews when dialogue moves between talking, drawing and modelling. Reassessing subjects over time might also reveal differences between the groups involved. Our hypotheses are spelled out in the section which follows.

\section{Methods}

The methodology was based on three main research efforts: (a) the pioneering work of Nussbaum and Novak (1976) and Nussbaum (1979) on children's cosmologies; (b) the early work of Napier (1956) and Elliot and Connolly (1984) into the dexterity of the human hand; and (c) the more recent research of Chien et al. (2010), Lin et al. (2002) on classification of children's hand movements. The procedure utilised Piagetian semi-structured interviews based on an extensive Interview Guide with three media of expression affording 
multi-modal responses: verbal language, drawing and play-dough modelling. The interviews investigated children's concepts of the Motion of the Earth through observation of changes in the shadow of a shadow stick, followed by drawing the motion of the Earth. Children also observed the Moon in daylight and traced the phases of the Moon over a month. The children then drew and modelled the shape of the Earth, Sun and Moon. The concept of "Identity with Earth" was also explored by drawing and using models of "Self" and "a Friend on the other side of the world"; and gravity, explored by investigating the trajectory of dropped and thrown balls, and falling water, providing further insights into their Earth Shape notions. Finally, children modelled the motion of the Earth, Sun and Moon using their own models (see Bryce and Blown 2006, 2007, 2012, 2013; Blown and Bryce 2006, 2010, 2012).

Three important elements of the interview technique are illustrated in the first protocol extract given in Sect. 6. Firstly, in the case of Earth Shape, an analogy was used to distinguish between spherical- and disc-like shapes. Secondly, in the case of Sun Shape modelling, we avoided asking questions while drawing or modelling was in progress so as not to disturb children. It has been found through experience (as in this case) that children tend not to engage in verbal dialogue while deep in thought during other modal activities such as modelling. However, this is countered by the need to know what the child is doing/thinking at each stage. Hence, a balance has to be struck between allowing thought to flow freely, and knowing what is going on in the child's mind. Thirdly, as in the example from Moon Shape modelling, drawings were used to cross-reference shape concepts from other modalities by having each child's drawing of the shapes of the Earth, Sun and Moon nearby to refer to. This was usually used when modelling the phases of the Moon, when children were encouraged to replicate in play-dough the shapes that they had described verbally and drawn earlier in the interview. This procedure also has its pros and cons. On the one hand, modelling phases can give children clearer insights into the scientific nature of phases as semi-spherical areas of the Moon visible from the Earth because of their illumination by sunlight. On the other hand, modelling phases could reinforce cultural ideas such as that the Moon really does have different physical shapes at different times of the month (particularly relevant in China, and Māori culture in New Zealand where the lunar calendar continues to be used for cultural festivals, and traditional activities such as sowing and harvesting crops, and fishing).

\subsection{Settings and Samples}

The interviews took place in local school and kindergarten settings in Featherston, Wairarapa, New Zealand (NZ) (2004-2005), and Changchun, Jilin, China (2005-2006), where the researcher (2nd author) initially spent considerable time periods with the staff and pupils, and the local communities concerned, to become familiar with their circumstances and to be known to the volunteer subjects taking part. During the interviews, the researcher spoke (in English) to the children taking part, his words being translated into Chinese in Changchun. Subjects' replies (in the case of the Chinese subjects, via the translator) were recorded and later transcribed for analysis. The subjects were asked to make drawings with paper, pencils and felt pen, and models with play-dough provided for the purpose. Drawings and models were retained/photographed for subsequent analysis. Verbal interviews, drawing sessions and play-dough modelling were recorded on audiotape, and the latter were also recorded on video tape which afforded analysis of hand movements. There were 360 participants in all, age 3-13, including 294 from NZ (158 boys and 136 girls) and 66 from China ( 34 boys and 32 girls). Of these, 36 children from 
NZ and 36 from China took part in longitudinal cross-cultural and cross-age comparisons; the subjects being matched on socio-economic background with approximately equal numbers of boys and girls in each group, and a similar control group for the follow-up studies (NZ: 2005; China: 2006).

As described previously, the research was based on multi-modal, one-to-one, Piagetian semi-structured interviews with Socratic dialogue conducted along ethnographic research principles. The participants were selected by class teachers who matched children according to general ability, socio-economic background, age, ethnicity and gender to achieve typical cross sections of age groups in each school community and culture. Although all responses were recorded and analysed, space precludes full reporting. The verbal protocols, drawings, play-dough models and modelling selected for comment are typical examples of their age group.

\subsection{Data Analysis, Categorisation and Coding}

The data were gathered with the help of an extensive Interview Guide, extracts of which are reported in the protocols (see also Bryce and Blown 2012; Blown and Bryce 2012). The second author did the initial coding of children's responses using the Cosmological Concept Categorisation Scheme developed by the authors. This had previously been independently assessed periodically by two astronomy educators from Carter National Observatory, Wellington, New Zealand (see Bryce and Blown 2012; Tables 1 and 2 below). The same coders were invited to independently code interview audiotapes, children's drawings, photographs of play-dough models and video protocols representing $12 \%$ of the comparative sample to provide measures of independent coding reliability. These were reviewed to achieve consensus and identify discrepancies with a resulting inter-coder agreement of 92-96 \%; Cohen's kappa $\kappa=.92-.94$.

Operational guidance regarding the pursuit of each of the research questions in turn is included in Sect. 6.

Additionally, and with respect to RQ2 (a) in particular, an attempt was made to quantify children's dexterous skill as they modelled shapes and motions, which we termed hand motion skill and motion concept skill, respectively. Our reasoning had been that dexterous skill in modelling concepts could be an exemplar of concepts as skills and simulations as hypothesised by Barsalou (2003) and that the elements of hand motion skill for modelling both shape and motion of ESM could be placed on an ordinal scale from least difficult to most difficult to perform. However, this proved to be much more difficult than originally anticipated (and ultimately unsuccessful in relation to the research questions pursued here), partly because our analysis was based on observation of video tape data stored on DVD rather than on more sophisticated techniques such as computer modelling capturing hand motion in image sequences (see Chien et al. 2010; Lin et al. 2002). For example, when attempting to measure hand motion skill, we were unable to capture rapid fine movements during shape modelling-motions which enabled older children to make spherical shapes with minimal gross hand motions such as rolling, confounding quantification based on summing observable gross motions (such as rolling between hands, rolling to and fro on desk, circular rolling on desk). And the full range of hand motions when modelling rotation and revolution simultaneously (some of which were hidden from view) was virtually impossible to quantify objectively into an ordinal scale of motion concept skill (see Jaimee protocol below).

To counter these difficulties with quantitative results, we decided to report the gross hand motions in qualitative form in selected protocols to at least yield a sense of the skills 
Table 1 Shape modelling concept categories

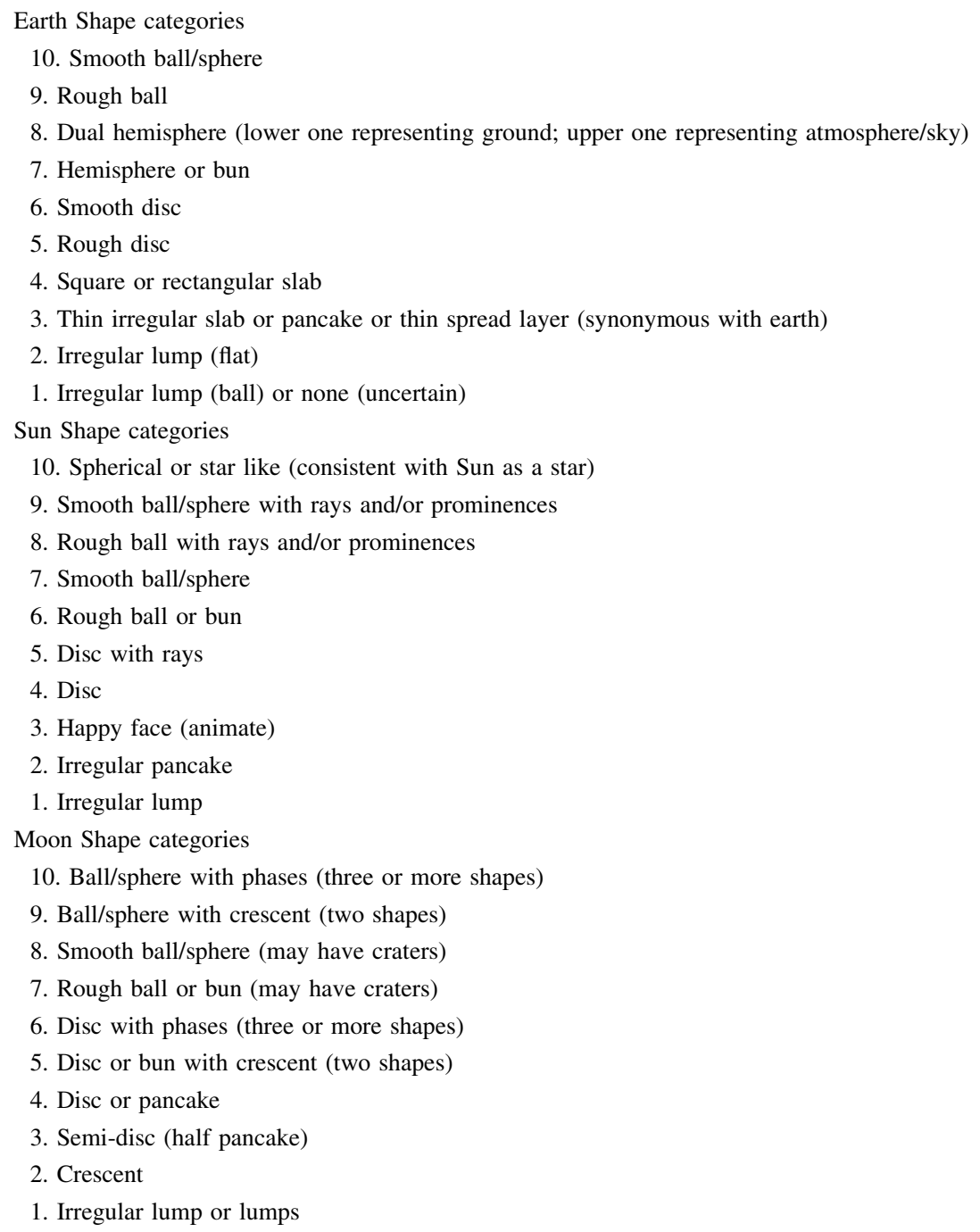

being used in each case. The quantitative results were limited to categorising the highest concept held by the child in each element in each modality on a ten-point ordinal scale from least scientific to most scientific for each concept: ESM Shape and ESM Motion. Thus, rather than classifying (categorising) the process of dexterous-concept skills being used to create a shape or motion, the concept categorisation scheme was used to classify the final product of the interview, drawing or modelling activity. The KolmogorovSmirnov two-sample test at an alpha level of .05 was then used to compare the means of groups based on the classification of children's concepts.

The evidence from the current studies strongly supports the proposition that children's cosmologies are dynamic in the sense of being in a constant state of flux influenced by 
Table 2 Motion modelling concept categories

\author{
Earth Motion categories \\ 9. Rotates on axis and revolves around the Sun \\ 8. Rotates on vertical or tilted axis (spherical) \\ 7. Revolves around Sun (but does not rotate) \\ 6. Rolls on horizontal axis (spherical) \\ 5. Rotates on axis (disc) \\ 4. Moves continuously in some way; e.g. across sky \\ 3. Moves intermittently; e.g. only at night-time; rolls to and fro \\ 2. Stationary \\ 1. Uncertain \\ Sun Motion categories \\ 8. Rotates on axis (spherical) \\ 7. Revolves in some way \\ 6. Rotates on axis and revolves around Earth \\ 5. Revolves around Earth \\ 4. Stationary \\ 3. Moves in some way; e.g. rises and sets; moves across sky; rotates (disc); rolls \\ 2. Animate; e.g. follows us; moves behind clouds \\ 1. Uncertain \\ Moon Motion categories \\ 8. Rotates on axis and revolves around Earth \\ 7. Revolves around Earth \\ 6. Rotates on axis (spherical) \\ 5. Moves with Earth as Earth revolves around Sun \\ 4. Moves in some way; e.g. rises and sets; moves across sky; rotates (disc) \\ 3. Stationary \\ 2. Animate; e.g. follows us; moves behind clouds \\ 1. Uncertain
}

interaction of everyday and scientific ideas stimulated by questioning and Socratic dialogue, and supported by multi-modal opportunities (drawing and play-dough modelling). The present analysis therefore merited reconsiderations of comparable data from the perspective of non-representational memory that ideas are dynamic and multi-modal, actively created at the point of recall. Skill in creating static and dynamic concepts such as the shape and motion of the Earth, Sun and Moon in multi-modal formats will increase with age so; in line with our previous findings concerning developmental trends in China and New Zealand, we hypothesised that:

H1 The dynamic structure of memory and concept creation will be similar in both genders and common across cultures [NZ boys $v$. NZ girls; China boys $v$. China girls; NZ v. China; NZ European $v$. NZ Māori].

H2 Repeated interviews in this longitudinal study will lead to enhanced modelling skills and/or more advanced shape and motion concepts. 


\section{Results and Discussion}

In this section, we present a number of extracts from the interview protocols to indicate the extent of the support for the research questions and hypotheses stated earlier. There were numerous supporting examples throughout the sample and the extracts chosen for illustration and presented below are broadly representative of age, gender and ethnicity (twelve consisting of 5 Chinese Han, 4 New Zealand European and 3 Māori). These reveal the switching taking place between modalities as children are questioned about the shape and motion of the Earth, Sun and Moon. The sample numbers from which these exemplars were drawn are larger than in many similar studies, allowing confidence for our conclusions.

The qualitative findings are grouped by research question, RQ1 through RQ4, and these are followed by the numerical data indicating the testing of the two hypotheses. The first protocol, that of Zhang Lan Ge (a 7-year-old female of Han ethnicity in Junior Primary School in China), is more or less complete and shows the structure of the interview with few interruptions. This includes details of the hand movements used to model shape and motion. We have concluded each of the extract analyses with a short discussion relevant to the aspect in question, thereby enabling the final discussion to stand back from the detail and be more comprehensible.

\subsection{Multi-Modal Thought in Tune with Edelman's Re-Entrant Signalling}

Is there any evidence that thinking involves mutually supportive, self-correcting and simultaneously adjusting processes instigated by different modalities-all the time, naturally, in accord with Edelman's re-entrant signalling? (RQ1 a). Confirming indicators: Child refers to all three modalities during interview with a variety of different responses from each modality requiring active integration by the child. Self-correcting processes are reflected in change of shape or modification to motion in response to Socratic dialogue. Disconfirming indicators: Child only repeats responses in same modality as previously used, no self-correction apparent. The following examples illustrate how children adjust their thinking-sometimes within a particular mode, often when they switch between modes.

\subsubsection{Zhang Lan Ge (China: Han: Female: Age 7 years 0 months: Junior Primary School)}

\section{R. Make the shape of the Earth with the green play-dough.}

R. [I give child an irregular lump of play-dough about 40 to $50 \mathrm{~mm}$ in diameter (but not spherical; to encourage creative modelling); similar 'lumps' of play-dough being used for all modelling of ESM.]

C. (Makes shape of Earth by gripping, pressing between hands, moulding between hands, circular rolling between hands, patting, pressing on desk, and circular rolling on desk).
R. What shape have you made there?
C. Round.
R. Round like a ball, or round like a pancake?
C. A ball.
R. [Earth spherical (people live inside): Earth Shape Category 7: see Table 1]. 
(See also Earth Shape Categorisation Scheme in Bryce and Blown (2013): Category 7: Earth Shaped like a ball cut in half: Ground and Sky inside the Earth: people live "inside" transparent dome of the Earth).

Note that Earth Shape Categories 7 and 8 are similar; both have people living inside the Earth on the flat surface (Ground) of a truncated sphere, but Category 7 has the Sky within the upper dome, whereas Category 8 has the Sky outside the dome as Space. Zhang's initial responses could also be interpreted as a "counter example" or "disconfirming indicator" of the RQ. From a single-modality perspective, Zang's model appears to be spherical, but her verbal responses and drawings reveal that she believed that people lived inside the Earth.

\section{Modelling the Shape of the Sun}

R. Make the shape of the Sun with the red play-dough.

R. [I give child a lump of play-dough.]

C. (Makes shape of Sun by gripping, pressing between hands, pressing on desk, circular rolling on desk, squeezing with fingers, and moulding on desk to form sphere; then pinching equatorial edge to form spikes/rays. Forms some of the latter by pinching pieces off of ball, horizontal rolling small piece of play-dough on desk, then re-attaching as rays).

R. What are the spiky bits?

$C$. (Does not respond-concentrating on modelling rays).

$R$. [I wait until child has completed modelling].

R. What are the spiky bits?

C. The sunlight.

R. [Sun Model is spherical with rays: Sun Shape Concept Category 7: see Table 1]. Note that here the child appears to have switched off the verbal language modality for a while in favour of giving maximum attention to the modelling modalities. Although at first sight a "counter example", the verbal language mode could be activated by the intervention of the researcher if he was prepared to interrupt the child's concentration on modelling rays as in the final question.

\section{Modelling the Shape of the Moon}

R. Make the shapes of the Moon with the yellow play-dough.

R. [I give child a lump of play-dough.]

$R$. [I introduce child's drawing of Moon for cross-reference].

$R$. You drew two shapes of the Moon (I indicate child's drawing). If you can make one with that (I hand child a lump of yellow play-dough; and one with that (I give child a second lump of yellow play-dough).

C. (Makes first shape of Moon by breaking play-dough into smaller pieces twice; then rolling horizontally between hands; then rolling on desk; to create a cylindrical shape).

$\boldsymbol{R}$. What shape have you made there?

C. A banana.

R. What shape have you made the other one?

$C$. (Makes second Moon Shape by circular rolling between hands).

R. Is the other one like a ball?

C. Yes.

R. [Moon Model ball plus crescent: Moon Shape Concept Category 7: see Table 1]. 
Modelling the Motion of the Earth, Sun, and Moon with play-dough Models

R. [The play-dough models of the ESM remain from the Shape study].

$R$. Does the Earth move?

C. Yes.

$R$. Show me how the Earth moves?

C. (Indicates spinning motion).

R. It spins?

C. At night it moves like this (models anti-clockwise motion looking down on North Pole) and in the morning it moves this way (models clockwise motion looking down on North Pole).

R. How long does it take to move like that (I model anti-clockwise motion)?

C. An hour

R. How long does it take to move like that (I model clockwise motion)?

C. An hour

R. [Highest Earth Motion Concept Category 4: moves continuously: see Table 2].

R. Does the Sun move?

C. Yes.

R. Show me how the Sun moves?

C. (Indicates a spinning and cross-ways motion).

$R$. It spins and moves across the sky?

C. Yes.

R. How long does it take to spin once (I model spinning motion)?

C. Half an hour.

$R$. How long does it take to move across the sky (I model crosswise motion)?

C. The Sun follows the car.

$R$. If this was a little person walking (I introduce model person) show me how the Sun follows?

C. (Shows Sun spinning and following with right hand; and person walking with left hand).

R. The Sun follows like that?

C. And at the same time it spins.

R. [Highest Earth Motion Concept Category 2: see Table 2].

\section{R. Does the Moon move?}

C. Yes.

R. Show me how the Moon moves?

C. It spins and also follows the car.

$R$. If that's a person (I introduce model person again) show me how the Moon follows?

C. (Shows Moon spinning and following with right hand; person walking with left hand).

R. [Highest Earth Motion Concept Category 2: see Table 2].

Although Zhang Lan Ge knows that the Earth rotates, she has yet to form a complete concept of the continuity of Earth Motion or associated concepts of time such as the concept and duration of a day. Consequently, her concepts of Sun and Moon Motion are grounded in a geocentric perspective, and her everyday observations of relative motion are 
translated into semi-animistic notions such as that the Sun and Moon follow us when we drive in a car. Modelling relative motion using play-dough models of the Sun and Moon together with small model people enabled children to share their ideas in a way that is much more meaningful than could be accomplished with verbal language or drawing. Although Zhang Lan Ge appeared to be aware of the rotation of both the Sun and the Moon and may have learned this from school or other sources of astronomical knowledge, it is more likely that she is making an analogy with Earth's rotation. Most children of her age have been introduced to elements of the heliocentric hypothesis such as the rotation of the Earth on its axis in a day and revolution of the Earth about the Sun in a year. Some may also have been taught of the Moon's revolution about the Earth in a month. But few would have been taught of the Moon's rotation in a month or the Sun's rotation (in 26 days). However, as we have argued elsewhere (see Bryce and Blown 2006, 2013; Blown and Bryce 2010) rather than being interpreted as "knowledge in pieces", Zhang Lan Ge's ideas should be thought of as genuine attempts to form a complete picture of the world (framework theory) from a variety of knowledge sources, both scientific and cultural, combined with her own observations.

\subsubsection{Jaimee (NZ: European: Female: Age 11 years 0 months: Senior Primary School)}

\section{Modelling Daytime and Night-time}

$R$. With the Earth, could you show me what daytime and night-time look like?

$R$. Imagine that the sunlight coming in the window is the real sunlight (shining on the Earth).

R. [I indicate sunlight shining on play-dough model Earth.]

$R$. Could you pick it up and show me daytime and night-time?

C. [Picks up play-dough model of Earth with right hand].

$\boldsymbol{R}$. Where's daytime if that's the Earth?

C. Daytime would be on this side (indicates sunlit side) and night-time would be on this side (indicates shaded side) because the Sun shines on one side at a time (rotates model Earth with both hands).

$\boldsymbol{R}$. How do we get daytime and night-time?

C. As the Earth moves around (rotates model Earth using both hands).

\section{Modelling the Motion of the Earth}

R. As it (the Earth) spins?

C. Yes.

R. How long does it take to spin once?

C. A day (rotates model Earth with right hand).

$R$. Does the Earth move in any other way?

R. [Earlier in her observational astronomy session (observing the motion of a shadow in sunlight) Jaimee had said that the Earth rotated and orbited the Sun, modelled it rotating and revolving, and drew it rotating in a day and revolving around the Sun in a year. I seek to clarify to what extent she has changed her mind and why].

C. [Indicates partial orbit around Moon with right hand holding model Earth].

C. Oh-it doesn't (move in any other way)! It spins! (returns to rotating model Earth in right hand then both hands).

$R$. [Child self-correcting].

$R$. It doesn't (move in any other way)? 
C. No (continues to rotate model Earth in right hand).

$R$. It doesn't move around the Sun-or anything like that?

R. [Recalling child's earlier statement that the Earth revolves around the Sun I use Socratic dialogue to jog her memory.]

C. I think so (stops rotating model Earth with right hand).

R. Does it?

C. Yes (moves model Earth to proximity of model Sun).

R. Can you show me how the Earth moves then?

C. [Models Earth rotating on axis with right hand and going around the Sun with right arm and wrist while holding Sun stationary in left hand].

R. [To see Earth Motion more clearly I offer to hold the model of the Sun].

R. I'll hold the Sun: you show me the Earth moving.

C. [Models Earth rotating on axis with both hands and going around the Sun].

$R$. [Active integration by child].

R. It's spinning and going around the Sun?

C. Yes.

R. How long does it take to go around the Sun once?

C. A year.

R. [Highest Earth Motion Concept Category 6: see Table 2].

Jaimee's understanding of the motion of the Earth encompasses the main scientific concepts of rotation, revolution and associated concepts of time (day and year). The sequence where her hands were indicating a partial orbit of the Moon while her words were weighing up the pros and cons of rotation versus revolution is revealing in that they show the two-way interplay between mental conceptions and physical actions argued by Edelman (2005). They also show the power of gesture ${ }^{4}$ in communicating ideas and that "... gestures and speech are most appropriately regarded as two sides of a single underlying verbal-gestural process of constructing and presenting meanings" (McNeill 1992, p. 24; see also Gallagher 2005). Based on these theories, a possible explanation would be that while modelling the Earth rotating, Jaimee's long-term memory (nonrepresentational according to Edelman) was activated by the open-ended question: Does the Earth move in any other way?

This may have caused her to recall the previous context in which she modelled the Earth rotating and revolving. This triggered neural pathways enabling concept creation which were transmitted to motor areas capable of producing motions by the hands to model the concept. Once the hands started to move, there would be feedback through the motor system to the neural pathways to compare the physical motion with the mental conception (dynamic concept) of the motion (see Edelman 2005). This would explain Jaimee's exclamation: Oh-it doesn't! (move in any other way) It spins! However, Jaimee realises that this explanation is incomplete and does not match her gesture (partial orbit around the Moon) or her long-term memory (from the earlier interview) that the Earth rotates on its axis and revolves around the Sun. The second question (It doesn't move around the Sun or anything like that?) focuses on the conflict and causes Jaimee to resolve the mismatch between concept and action. At this stage, Jaimee pauses to think (I think so) and ceases to rotate the model Earth. Then, convinced by feedback from comparison with long-term memory, she realises that the Earth not only rotates but also revolves-but not around the

\footnotetext{
4 Although we recognise the value of gesture and facial expressions in communication we did not include them as major modalities for reasons of privacy. In cooperation with school head teachers, we arranged the video recorder so that in general it only showed children's hands against a background of the desktop. This excluded gestures such as hands wide apart (which could have included facial features and revealed the identity of the child).
} 
Moon around the Sun. She responds Yes and returns to modelling the Earth rotating on its axis and revolving around the Sun.
Modelling the Motion of the Sun
$R$. Does the Sun move?
C. No.
R. [Most children of this age consider the Sun to be stationary].
R. [Highest Sun Motion Concept Category 8: see Table 2].

\section{Modelling the Motion of the Moon}

$R$. Does the Moon move?

C. Yes.

R. How does the Moon move?

C. Around the Earth.

$R$. Can you show me that as well then?

C. [Models Moon revolving around Earth first using right hand, then using both hands].

$R$. How long does it take to go round the Earth once?

C. A day.

R. Does the Moon spin (I model)?

C. Yes.

R. How long does it take to spin once?

C. A year.

R. How long to go round the Earth?

C. Oh no! That's a year. It spins once in a day.

$R$. [Child self-corrects].

R. [Highest Moon Motion Concept Category 7: see Table 2].

Although Jaimee knows and can model the main Moon Motions (revolution and rotation), her knowledge of associated concepts of time is inaccurate as shown by her statement: $C$. Oh no! That's a year. It spins once in a day in response to the researcher's: $R$. How long (does the Moon take) to go round the Earth?

Nevertheless, her responses indicate the self-correcting processes that are a characteristic feature of what must follow from Edelman's (2005) re-entry signalling.

\subsubsection{Lü Shi Hao (China: Han: Male: Age 8 years 8 months: Junior Primary School)}

\section{Modelling the Shape, Nature and Structure of the Moon with Play-dough}

R. Could you make the shape of the Moon?

C. [Commences to mould play-dough into ball shape].

$R$. You made (drew) four Moon Shapes (I show child drawing)_you can make all of these?

$R$. [I hand child four lumps of yellow play-dough].

C. [Makes 1st Moon Shape-a thin crescent-by circular rolling between hands to form a sphere; then pressing between hands to form disc; then tearing segment from disc].

C. [Makes 2nd Moon Shape-a crescent-by repeating the above process].

C. [Makes 3rd Moon Shape-a thick crescent-by rolling into ball by circular rolling between hands; then squeezing and teasing to form disc; then moulding into semi-sphere]. C. [Makes 4th Moon Shape-a ball-by collecting together discarded pieces of playdough and rolling and moulding them between hands]. 
$R$. [Child appears to have completed modelling task so I ask:]

$\boldsymbol{R}$. What shape have you made there (I indicate ball-shaped model Moon)?

C. [Considers].

R. Is that like a ball or like a pancake?

C. [Commences to flatten ball by pressing on desk with both hands].

R. Like a pancake?

C. Yes (continues to flatten model-then flips over and presses on reverse side to form disc).

R. [Moon Model disc with phases: Moon Shape Concept Category 6: see Table 1].

Lü Shi Hao's modelling of Moon shapes demonstrates that, in common with Chinese children of his age, he was acquainted with two coexisting concepts of the Moon: the cultural Moon (yuè liang) which changes shape each month and is the basis of the Chinese lunar calendar, and the scientific Moon (yuè qiú) which is always spherical. This difference is shown by Lü Shi Hao modelling the scientific full Moon Shape as a ball; then modelling the cultural full Moon Shape as a disc or pancake (bing); the crescent-shaped phases being associated with both concepts, i.e. with the scientific Moon as sections of a sphere, and with the cultural Moon as sections of a disc as seen in the night sky. The sudden change from modelling the Moon as ball shaped to modelling it as a disc was triggered by the question sequence:

$R$. What shape have you made there (indicating ball-shaped model Moon)?

$R$. Is that like a ball or like a pancake?

This appeared to activate a process of self-correction to resolve the cognitive conflict of matching the shape of the full Moon to phases modelled as sections of a disc. The ready transformation from ball to disc afforded by play-dough suggests active integration of shape concepts based on personal observation, cultural influences and teaching. The final model of a disc-shaped Moon with matching phases (a variety of sections of a disc) represents the child's attempt to integrate differing inputs from multiple modalities.

\subsection{Non-Representational Nature of Memory, and Concepts as Generalities or Universals}

Are there grounds for believing that "memory is non-representational and concepts are the outcome of the brain mapping its own perceptual maps leading to generalities or 'universals", (Edelman 2005, p. 104)? Confirming indicators: Child switches between 1D, 2D and 3D mental constructions of ESM shapes and motions. Disconfirming indicators: No switching evident for either shape or motion concepts, either immediately at that juncture in the interview or subsequently. Here, the examples illustrate the interplay of all three modalities and in doing so lending clarification to the meaning of ideas. They also show that children can hold both everyday and scientific versions of concepts simultaneously, or may switch between them.

\subsubsection{Alistair (NZ: European: Male: Age 10 years 9 months: Senior Primary School)}

\section{Modelling the Shape of the Earth}

\section{$R$. Can you make the shape of the Earth with the green play-dough?}

C. [Models shape of Earth by gripping play-dough with right hand; pressing between hands; moulding between hands; and gentle pressure with right, left and both hands].

R. [Model is spherical: Earth Shape Concept Category 10: see Table 1]. 


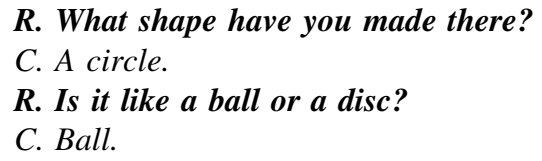

Alistair's use of the term "circle" to describe sphericity is common for children of his age. Although the use of cultural models is questionable (see Bryce and Blown 2006), we justify their use here where children have already created their models and are matching them to an artefact (wooden disc or tennis ball) rather than selecting a shape from a range of models with no modelling. It does however raise the question of how the concept of circularity is created (see Fig. 1).

\section{Modelling the Shape of the Sun}

$R$. (Can you) make the shape of the Sun with the red play-dough?

C. [Models shape of Sun by gripping play-dough with his right hand; pressing between hands; moulding between hands; and pinching rays/spikes.]

R. [Model is a smooth ball with rays: Sun Shape Concept Category 9: see Table 1].

$R$. What are the points?

$C$. The Sun's got thermo-nuclear energy.

R. What shape is that then (indicating model)?

C. It's a circle.

$R$. Is it a circle like a ball or a circle like a disc?

C. Like a ball.

$R$. Do the spikes come out of it all over or only on the edge?

C. On the edge.

The source of the Sun's energy was discussed earlier in the interview when children were asked general questions such as Tell me about the Sun? These were not probed in depth here; but it is noted that, although Alistair is aware of the nature of the Sun's radiant energy, he is unable to fully translate this concept. He described the Sun as having rays and drew it with spikes around its circumference, but he did not realise that his pictorial representations were incomplete and that the rays emanated not from the $2 \mathrm{D}$ edge of a circle but from the 3D spherical surface of the Sun. This example illustrates the advantage of triangulating concepts from different media and modalities. It also highlights the need to use three-dimensional models or visual aids such as computer graphics, videos, DVDs of the Earth, Sun and Moon when teaching astronomy so that children can build more complete concepts of the shape, structure and nature of these heavenly objects as planets, stars and natural satellites rather than circular objects on a board or in a book. No matter how well illustrated, the latter remain two-dimensional.

\section{Modelling the Shape of the Moon}

$R$. Can you make the shape of the Moon with the (yellow) play-dough? And if you think that the Moon is different shapes-like you have drawn (I indicate child's drawing) then you can make these different shapes.

C. [Models 1st Moon Shape-a crescent-by gripping and squeezing with both hands; pressing between hands; rolling between hands; moulding between hands; breaking the play-dough lump into smaller pieces; and gripping and curving into a crescent].

$R$. Is that (I indicate model of crescent Moon) the same as is this one over here (I indicate drawing of crescent Moon)?

C. Yes. 
C. [Models 2nd Moon Shape-a disc-by shaping using one hand as an anvil; pressing and squeezing with fingers; moulding and shaping with fingers; squeezing and teasing into a disc; and patting with hands].

R. What shape is that there?

C. Full.

$R$. Is that shape there (I indicate child's disc-shaped model of Moon) like this (ball) or that (disc)?

R. [I show child a ball and a disc to clarify].

C. A disc [selects disc].

R. That's this one (I indicate drawing of full Moon)?

C. Yes.

R. [1st Model is crescent shaped; 2nd disc: Moon Shape Concept Category 4: see Table 1].

Direct comparison between the children's models and their earlier drawings usually enabled conceptual conflict to be reduced leading to a clearer understanding of shape. However, Alistair believes the Moon to be disc shaped, and models it as such. His drawing of the full Moon as a circle being 2D is ambiguous, but his selection of the disc shape is clear-cut. He has yet to develop the conceptual skill (see Barsalou 2003) that will enable him to reason that if the Earth and Sun are spherical then it is likely that the Moon is also spherical. His notion of the Moon being disc shaped is based on a 2D perspective grounded in the concept of circularity rather than a 3D view of sphericity (see Fig. 1). This extract from a typical child's interview illustrates the interplay of all three modalities in clarifying concepts and building meaning.

\subsubsection{Li Xin (China: Han: Female: Age 9 years 9 months: Senior Primary School)}

\section{Modelling the Shape of the Sun}

$R$. If you can make the shape of the Sun with the red play-dough?

C. [Makes ball-shaped model of the Sun by gripping, pressing between hands, circular rolling between hands, and passing between hands].

$\boldsymbol{R}$. What shape have you made there?

C. Round.

R. Is that round like a ball or round like a pancake?

C. Like a pancake.

R. It doesn't look like a pancake?

$C$. The real Sun is like a pancake.

R. Can you make it the shape of the real Sun?

C. \{Picks up spherical model of Sun and presses to disc using palm and back of right hand using left hand as anvil, and finally patting with right hand].

R. [Final Sun Shape Model is a disc: Sun Shape Concept Category 4: see Table 1].

Here Li Xin appears to be differentiating between the spherical "scientific" Sun (as taught by teachers in school) and the disc-shaped "real" or "everyday" Sun (as observed at sunrise and sunset in the "real" world). Possibly, these two concepts coexisted in some form (such as mental imagery) in the child's memory-or the capacity to form them coexisted in the non-representative neural substrate from which concepts are created by both conscious and unconscious processes and mental skills (see Barsalou 2003; Edelman 2005). Note that Li Xin appears to represent a "disconfirming" example in terms of the verbal modality in that despite the fact that she modelled the Sun as ball-shaped she 
describes it as "round" and "like a pancake" by analogy. However, although her verbal description remains the same, her model switches from ball-shaped to disc-shaped.

\subsubsection{Renee (NZ: Māori: Female: Age 12 years 3 months: Senior Primary School)}

\section{Modelling the Shape of the Earth}

$R$. Can you make the shape of the Earth with the green play-dough?

C. [Models shape of Earth by gripping and squeezing with right, left and both hands; pressing between hands; moulding between hands; pressing on desk; pressing with fingers; rolling between hands; patting between hands; and gentle pressure with both hands].
R. What shape are you making there?
C. Round like a ball.
R. [Final Earth Shape Model is spherical: Earth Shape Category 10: see Table 1].

\section{Modelling the Shape of the Sun}

$R$. (Can you) make the shape of the Sun with the red play-dough?

C. [Models shape of Sun by gripping play-dough with right, left, and both hands; pressing between hands; moulding between hands; and passing/patting between hands].

$R$. What shape is that (I point to child's model)?

C. Round like a ball as well.

R. [Final Sun Shape Model is a smooth ball: Sun Shape Category 7: see Table 1].

\section{Modelling the Shape of the Moon}

R. Make the shape of the Moon.

C. [Commences to model Moon by gripping and squeezing with left, right, and both hands].

$R$. Now you made (drew) several shapes of the Moon-you made (drew) all of these different shapes (I show child her drawing of Moon Shapes)_if you want to make all of them you can (full Moon and five phases)-or you can make a few of them-if you need more play-dough I can give you more-if you want to break it up and make all of them I can leave the shapes (drawing) there (alongside the play-dough modelling sheet) if you want to refer to them.

C. [Breaks play-dough into smaller lumps and creates lunar phases by pressing playdough on desk with right and left hands; pressing between hands; gripping and curving into a crescent; curving crescent pressing with fingers on desk; pressing/squeezing with fingers of left, right and both hands; moulding and shaping with fingers of right and left hands; rolling between hands; moulding/shaping with fingers; and moulding hemisphere.].

$R$. Just put them down here (I indicate modelling sheet) when you are ready.

C. [Models 1st Moon Shape-a crescent].

$R$. They can be anywhere on there (I indicate the modelling sheet)-they don't have to be all in a line (as in child's drawing).

C. [Completes 1st crescent Moon Shape and places it on modelling sheet].

$R$. If you need any more play-dough just say so.

C. [Models 2nd Moon Shape-a semi-sphere-and places it on modelling sheet].

R. Good (conversational-not meant to imply evaluation). 
C. [Models 3rd Moon Shape-a sphere-and places it on modelling sheet].

R. Good (again conversational).

C. [Models 4th Moon Shape-another crescent-and places it on modelling sheet].

R. Right-are you happy with that?

C. Yes.

R. [Final Moon Shape Ball with phases: Moon Shape Category 9: see Table 1].

Modelling phases was thought to help older children to visualise sections of a spherical body like the Moon.

\section{Comparison between Shape Drawing and Shape Models}

$R$. So that shape (I indicate child's model of Earth) is more or less the same shape as you drew there (I indicate child's drawing of the Earth)?

C. Yes.

R. And that's a ball shape?

C. Yes.

$R$. And the Sun-that's the Sun Shape there (I indicate child's model of Sun and drawing of Sun)—another ball shape?

C. Yes.

R. What shape is that one there (I indicate child's drawing of full Moon)?

C. That's a round one (indicates drawing of full Moon with right hand and model of full Moon with left hand].

C. That's a crescent [indicates model of crescent Moon with left hand].

$C$. And that's a half [indicates model of quarter Moon with left hand].

R. Is that basic shape like a ball as well (I indicate model of full Moon)?

C. Kind of (gestures ball shape by cupping hands)—hold it!

C. [Picks up play dough model of full Moon and rolls between hands in a circular motion].

R. Is it like a ball?

C. [Continues to roll model between hands].

R. Is it meant to be a ball?

C. Um-yes.

C. [Continues to shape model of full Moon].

R. It's meant to be a ball?

C. [Places model back on modelling sheet].

R. So they're all sections of a ball?

C. Yes.

R. The basic shape is a ball?

C. Yes.

Comparison between drawings and models helped to clarify shape concepts by comparing 2D concepts from the drawing media/modality with 3D concepts from the modelling media/modality, using the media/modality of verbal language to facilitate matching. Renee's use of gesture to visualise the full Moon also suggests comparison with some form of imagery in memory through the kinesthetic modality. Her persistence in trying to create a perfectly spherical model of the Moon also suggests some form of two-way comparison taking place between a concept in her mind and the physical model in her hands in real time with more or less instant feedback (suggesting dynamic change at both ends of the process, i.e. changes to the concept in mind shaping changes to the concept in hand and vice versa: a two-way dynamic process incompatible with mental model theory, better explained by Barsalou/Edelman theory and Donaldson (1978), than by mental models). Matching the drawings and models also helped 
to clarify that the phases of the Moon do not constitute physical changes but are cultural explanations for the apparent changes in shape as a result of varying degrees of illumination.

\section{Modelling Ground and Sky}

$R$. If this was the Earth (I indicate child's play-dough model of the Earth) where would the ground be?

C. Round here (cups right hand around model Earth just touching surface).

R. Where would the sky be?

C. Above (flattens hand a few $\mathrm{cm}$ above model Earth) - around it (moves hand tangentially over top of model Earth).

\section{R. All around?}

C. Yes.

Modelling the ground and sky gives insights into children's concepts of Earth as a planet in space habited by mankind. Renee realises that the ground is the surface of the Earth and the sky envelopes the Earth in the same way as the atmosphere.

\subsubsection{Supporting Evidence: Variations of the Root Concept of Circularity in Drawing and Modelling Earth Shape and Motion Concepts}

Support for the argument (RQ1b) that memory may be non-representational and that concepts may be considered as generalities or universals comes from evidence from interviews of a core concept of circularity underlying children's concepts of shape and motion. In keeping with Edelman's (2005) re-entry theory, this core concept informs 2D and 3D shape and motion concepts. We argue that shape concepts such as spheres, discs and cylinders are derivatives of the concept of a circle. Similarly, motion concepts such as rotation and revolution are derivatives of a circular twist and a circular path (orbit). There is also interplay (imagery) between 2D and 3D concepts of circularity best replicated by play-dough modelling where there are three axes of shape and motion available to the mind/hands (see Fig. 1).

\subsection{Memory as a Dynamic Process with Concepts Reflecting Deep Links between Perceptual Motor Skills and Higher-Level Cognition}

Is there any data to support the view that memory consists not in the storage and retrieval of symbols, but results from a dynamical process of activation whereby concepts reflect deep links between perceptual motor skills and higher-level cognition (c.f. Clancey 1999)? (RQ2a). Confirming indicators: Child demonstrates coordination between perceptual motor skills such as hand motion skill (utilised when modelling the shape of ESM), and higher-level cognition such as being able to describe and draw the shape of ESM. Conceptual stability and change is coordinated through re-entrant signalling between higherlevel cognition in the mind and different motor modalities representing concepts through speech, drawing and modelling. Disconfirming indicators: No evidence of the child changing his/her mind when describing, drawing or modelling ESM shape concepts. Responses remain fixed. The illustrations here show how children can change their minds, often suddenly, in the course of their explanations, which can be considered as reflecting the dynamism emphasised in the Edelman/Barsalou conceptions of what is taking place in the brain during recall (as opposed to the notion of simply retrieving a mental model). 
6.3.1 Casey (NZ: European: Female: Age 7 years 2 months: Junior Primary School)

\section{Modelling the Shape of the Earth}

$R$. Can you make the shape of the Earth with the green play-dough?

C. [Creates disc-shaped model of Earth by gripping play-dough to pick up and put down model with right hand, pressing - on desk; with fingers; and between hands; moulding between hands and gentle pressure with both hands].

\section{R. Is that it?}

C. [Crumples between hands then rolls in a circular motion on desk to create ball shape].
R. Oh-you've not finished?
C. I need to make it round.
C. [Continues to shape into sphere by circular rolling on desk and between hands].
$\boldsymbol{R}$. What shape have you made there?
C. A ball.
C. [Places model Earth on modelling sheet].
R. [Final Earth Shape Model is spherical: Earth Shape Category 1: see Table 1].

Casey unexpectedly crumpled her disc-shaped model Earth into an irregular lump and formed a ball shape. This change indicated that Casey had two competing concepts of Earth in mind; the flat Earth represented by the ground of everyday experience and the spherical Earth of science. The process triggering the transformation is unknown, but it was not the result of Socratic dialogue with the researcher: the child changing her mind without dialogue other than "inner speech" (c.f. Vygotsky (2012).

\section{Modelling the Shape of the Sun}

$R$. (Can you) make the shape of the Sun with the red play-dough?

C. [Creates ball-shaped model by gripping to pick up and put down with right hand; pressing between hands; moulding between hands].

C. [Breaks piece off of play-dough ball to make rays].

C. [Re-forms ball shape by moulding between hands and circular rolling on desk].

C. [Forms first ray by rolling between fingers and attaches to ball-shaped model].

C. [Prepares second ray then changes mind-removes first ray and crumples ball into irregular lump incorporating rays and leftover play-dough from ray forming lump].

R. OK? (Interrogative).

C. [Flattens lump on desk and moulds to form disc].

R. Is that it?

C. Yes.

C. [Places model Sun on modelling sheet].

R. [Final Sun Shape Model is a disc: Sun Shape Concept Category 4: see Table 1].

\section{Follow-up questions}
R. What shape have you made the Sun?
C. It's round.
R. Is that like a pancake, or like a ball?
C. A pancake. 


\section{Comparison between Shape Drawing and Shape Models}

$R$. Is that shape of the Sun (I indicate model) the same as you made (drew) there (I indicate drawing)?

C. [Considers]

R. This one (drawing) has spikes (rays) and that one (model) hasn't, has it?

C. I can't make spikes on it.

Casey initially made a ball-shaped model of the Sun by moulding the red play-dough lump between her hands. For some reason, she then decided to break a lump off of her already formed ball to provide material for the construction of rays (or "spikes" in children's vernacular - analogous to the spikes of a hedgehog or sea urchin—kina in Māori). Casey then re-formed what remained of her original ball shape into a sphere and proceeded to make rays to attach to the spherical model of the Sun. She attached the first ray successfully but in the middle of attaching the second ray suddenly changed her mind. She carefully removed the first ray, crumpled the spherical model, the rays, and the lump used to form the rays into a single lump, which she moulded to form a ball. Then, again unexpectedly, she appeared to change her mind for a second time and pressed the ball on the desktop to form a disc. These changes indicate that Casey had at least three concepts of the Sun in mind: one spherical without rays representing an approximation of the scientific concept; the second, spherical with rays as portrayed in many children's story books; and third, the disc-shaped everyday Sun as experienced at sunset and sunrise. Casey's explanation for abandoning her spherical model with rays (replicating her drawing) was that she couldn't create and attach rays to her satisfaction. This led her to revert to earlier everyday models of Sun Shape.

These rapid transformations - in the case of Earth Shape from disc to ball and in the case of Sun Shape from ball, to ball with rays, to ball, to disc-suggest that Casey had access to creative processes in memory (higher-level cognition, c.f. Clancey 1999) which enabled her to compare verbal descriptions from dialogue with the researcher, with previously constructed drawings, to form models in play-dough interactively utilising multiple modalities. This appeared to involve matching and evaluative processes (matching of shape, and evaluation of kinesthetic and fine motor skills used in the modelling process). So that, when the play-dough model did not meet the matching or evaluative criteria, it was rejected in favour of a more achievable model.

\subsubsection{Kane (NZ: Māori: Male: Age 8 years 11 months: Junior Primary School)}

\section{Modelling the Shape of the Earth, Sun and Moon with play-dough Modelling the Shape of the Earth (1)}

\section{$R$. Could you make the shape of the Earth with the green play-dough?}

C. Creates initial model of Earth by gripping play-dough lump; and pressing to pancake shape on desktop and between hands].

R. It's a kind of flat shape, is it?

C. [Refines shape to disc by pressing with fingers].

R. Like a pancake?

C. Sort of like a ball (gestures ball shape by cupping hands together).

$R$. It's meant to be a ball, is it?

C. No-it's like this (gestures concave curve with right hand indicating surface of large ball).

R. [Initial Earth Shape Model is disc: Earth Shape Category 6: see Table 1]. 


\section{Modelling the Shape of the Sun}

$R$. Can you make the shape of the Sun with the red play-dough?

C. [Models Sun as spherical by gripping; and circular rolling between hands and on desk].

R. What shape is your Sun?

C. A circle-like a ball.

R. [Sun Shape Model is spherical: Sun Shape Concept Category 7: see Table 1].

Modelling the Shapes of the Moon

$R$. Can you make the shapes of the Moon with the yellow play-dough?

C. [Models crescent Moon by gripping; rolling between hands then curving into crescent].

R. That's the crescent one?

C. Yes.

C. [Models full Moon by circular rolling between hands and on desktop]

R. What shape is this one?

C. Like a ball.

R. So the Sun's like a ball and the Moon's like a ball?

C. Yes.

R. [Moon Model is ball with crescent: Moon Shape Category 9: see Table 1].

\section{Modelling the Shape of the Earth (2)}

\section{R. What about the Earth?}

C. [Considers].

R. You've made that like a pancake there, haven't you (I indicate child's model)?

C. [Considers].

R. Is it like a ball or like a pancake?

C. Like a ball.

R. Why have you made it like a pancake then?

C. Because I was going to roll it up.

$R$. Were you? OK (I hand model to child).

C. [Folds pancake to half disc then squashes to rough ball.

R. You were still shaping it (before)?

$R$. [I try to determine why child changed mind.]

C. Yes (refines shape to ball by circular rolling on desktop and moulding between hands).

R. You weren't meaning that sometimes it looks flat, were you?

C. Yes.

R. But it's really like a ball?

C. Yes.

R. Earlier the child described and drew the Earth as a planet in space; with ground as surface and sky around; and "Self" and "Friend" on opposite sides.

R. [Final Earth Shape Model is ball: Earth Shape Category 10: see Table 1].

In Kane's case, transformation from a disc shape to a ball shape was initiated by comparison between his disc-shaped model of the Earth and his ball-shaped models of the Sun and Moon. Kane's protocol demonstrates the role of gesture as a valuable but relatively neglected modality. Here Kane used gesture to show the shape of the Earth in two ways: firstly to show that the Earth is ball shaped by cupping his hands together as if 
enclosing a sphere, and secondly by moving his hand and arm in an arc to show that the surface of the Earth is in the form of a gentle curve. Thus, the end shapes of models are only part of the story: it is necessary to consider all model shapes created by the child (utilising perceptual motor skills such as hand motion skill) together with any gestures of shapes and triangulate these with verbal dialogue and drawings to form a more complete picture of what may be going on in the child's mind as they attempt to share their concepts.

\subsubsection{Zhang Zhe (China: Han: Male: Age 8 years 11 months: Junior Primary School)}

\section{Modelling the Shape of the Earth with play-dough}

$R$. Make the shape of the Earth with the green play-dough.

C. (Breaks play-dough lump into smaller pieces).

C. (Makes shape of Earth like a ball by rolling between hands then flattens it to a disc by pressing with right hand on desk top).

$\boldsymbol{R}$. Oh-are you flattening it now?

C. Yes.

R. I thought it was like a ball?

R. Is it not like a ball?

C. The Earth is round like a ball but I thought I should shape it like my drawing.

R. Make it the shape that it really is.

C. (Shapes Earth as a ball by rolling between hands).

$\boldsymbol{R}$. What shape have you made there?

C. Round.

R. Like a ball?

C. Yes.

R. [Final Earth Shape Model is ball: Earth Shape Category 10: see Table 1].

Zhang Zhe unexpectedly (since he had described the Earth as ball shaped) pressed his spherical Earth into a disc using his right, left and both hands in succession. When asked why, he said he wanted his model to match his drawing that was circular in shape (see protocol). Following Socratic dialogue, he transformed his disc-like Earth firstly into a rough ball then into a smooth ball and repeated this process until he was satisfied that his model shape represented a spherical Earth. This change from modelling the Earth as ball shaped to disc shaped and then back to ball shaped illustrates the simultaneous interaction between multiple modalities, presumably through multiple neural pathways connecting cognition and motor control. The initial ball shape was created in response to a verbal request (language modality) which triggered some aspect of the subject's memory and resulted in his brain and motor system making a sphere (kinesthetic modality). Then some form of internal mapping compared the spherical model with the apparently flat circular shape of his drawing (although his drawing was on the desk he did not glance at it while modelling the Earth, so his reference to the drawing modality probably entailed imagery rather that perception). This caused him to change his mind and transform the ball shape to a disc shape by pressing between his hand and the desktop. This motion would have resulted in kinesthetic feedback to his motor system to confirm that the model now matched the drawing (in terms of the criterion of $2 \mathrm{D}$ circularity). However, the researcher's surprise (communicated in the verbal language modality) caused Zhang Zhe to reassess his model and to focus on the 3D rather than the $2 \mathrm{D}$ attributes (i.e. on sphericity rather than circularity). This resulted in a second transformation of the play-dough medium from disc shaped to ball shaped (by circular rolling between the hands), satisfying the new matching 
criteria between verbal description (round, like a ball), drawing (circular, like a ball) and play-dough modelling-kinesthetic modalities (spherical).

Note that although Zhange Zhe changed his mind while modelling the shape of the Earth, once he had settled on his final model he kept its shape constant while he modelled the motion of the Earth, Sun and Moon. Thus, although there were variations in the products of hand motion skill, from the perspective of motion concept skill there were no variations in shape: a "counter example" or "disconfirming indicator" of the RQ.

The processes described have been included as an example of multi-modal modelling. We agree that the psychological and physiological processes are probably commonplace in everyday activities, but it served as an exemplar in support of a multi-modal methodology. Switching between everyday flat Earth and scientific spherical Earth concepts and vice versa is a dynamic process which probably occurs frequently. However, capturing this process in action either spontaneously or as a result of Socratic dialogue is comparatively rare.

\subsubsection{Supporting Evidence: Correlation Graphs of Earth Shape versus Earth Motion in Three Modalities}

Support for the arguments (RQ1b and RQ2a) that rather than being representational, memory is a dynamic process with concepts reflecting deep links between perceptual motor skills and higher-level cognition comes from correlation studies plotting shape against motion concepts. For example, plotting the Cosmological Concept Categories for Earth Shape (in three modalities: interview, drawing, play-dough) against those of Earth Motion (also in three modalities) with selected participants from New Zealand $(n=36)$ and China $(n=36)$ : hence, $N=72$, revealed correlations of $r=.77 ; r=.74$ and $r=.72$, respectively. See Fig. 2 and the relevant numerical data shown below. ${ }^{5}$

As we noted at the end of the section reviewing recent ideas in cognition, there may be several factors underlying these relationships, such as how ideas about planetary shape and motion figure during instruction or in presentations which children come across in books and television, but one possibility relates to the now-demonstrated multi-modality of the sensory-motor system. Modelling motion scientifically and modelling shape scientifically may be pertinent reflections of that multi-modality. Our first consideration had been that the coherence among the shape and motion concepts might indicate that there is a deeper underlying structure (possibly founded on roundness, circularity and sphericity, as shown in Fig. 1). But, regardless of whether or not this is the case, the coherence of these

\footnotetext{
${ }^{5}$ Data from all 360 participants were reviewed and analysed in preparing this report, of which those from the longitudinal survey group were considered to be most informative from a modelling perspective because it was based on matched pairs and taken from the initial interviews. The scatter of ordinate values in the mid range of the distribution was attributed to a region of transition as children's concepts tended to become more scientific.
}

Earth Shape Play-Dough v Earth Motion Play-Dough: Pearson $r=.77$; significant @ $p<.05$.

Equivalent Spearman $r_{s}=.73 ; t(N-2)=9.06 ;$-level $=.0000 ; N=72$.

Earth Shape Interview v Earth Motion Interview: Pearson $r=.74$; significant @ $p<.05$.

Equivalent Spearman $r_{s}=.69 ; t(N-2)=7.90 ;$ p-level $=.0000 ; N=72$.

Earth Shape Drawing v Earth Motion Drawing: Pearson $r=.72$; significant @ $p<.05$.

Equivalent Spearman $r_{s}=.71 ; t(N-2)=8.46 ;$ p-level $=.0000 ; N=72$. 

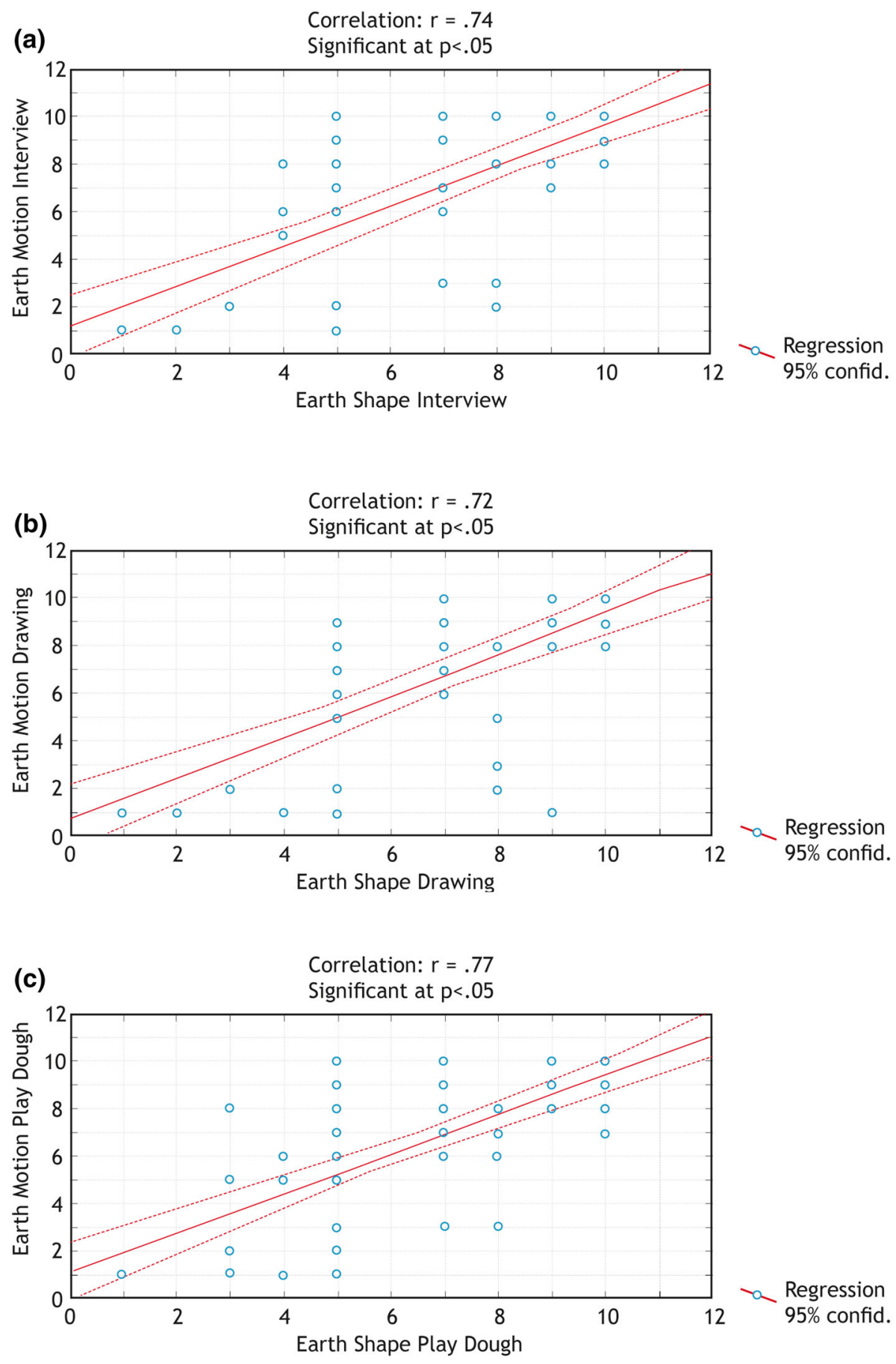

Fig. 2 a Correlation graph of Earth Shape Interview versus Earth Motion Interview. b Correlation graph of Earth Shape Drawing versus Earth Motion Drawing. c Correlation graph of Earth Shape Play-Dough versus Earth Motion Play-Dough 
conceptions does support the notion that children's ideas are both dynamic and multimodal.

These strong correlations between shape and motion concepts across modalities support the arguments of RQ1b (that memory may be non-representational and that concepts may be considered as generalities or universals) and RQ2b (that memory is a dynamic process with concepts reflecting deep links between perceptual motor skills and higher-level cognition). The correlations suggest that there is an underlying core concept of commonality between shape and motion of the ESM, such as the concept of circularity discussed above (shape concepts such as spheres and discs are derivatives of the concept of a circle; motion concepts such as rotation and revolution are derivatives of a circular twist and a circular orbit). The evidence suggests that if a child cannot describe, draw or model the Earth as a sphere (as a circle in 2D, or a ball in 3D), it is unlikely that they can visualise the Earth rotating (spinning) on its axis or revolving in a circular (or elliptical) orbit around the Sun. Whereas a child who knows that the Earth is spherical and can describe, draw and model a spherical shape, is better able to utilise dynamic imagery and higher-level cognition to imagine the Earth spinning and orbiting the Sun, and to model these processes using perceptual motor skills.

\subsection{Concepts as Simulators or Skills in Creating Images Consistently Over a Range of Modal Stimuli and Responses}

Is there any evidence in support of the contention that concepts are skills or simulators for constructing idiosyncratic representations (Barsalou 2003)? (RQ2b). Confirming indicators: Child refers to all three modalities during interview with similar responses from each modality (reinforced by repetition of performance; i.e. forming the same concept repeatedly within and across media). Thus, illustrating coherence of images of ESM Shape and/ or Motion mediated by skill in image creation, and cognitive ability in static and dynamic mental simulation. Disconfirming indicators: Child refers to the immediate modality only and responses echo previously configured shapes and presentations without interconnecting thoughts. These examples illustrate the importance of children striving for consistency among their thoughts, whether-for at least the duration of the interview-these thoughts result in improved scientific thinking, or the maintenance of alternative frameworks.

\subsubsection{Linda (NZ: European: Female: Age 7 years 2 months: Junior Primary School)}

\section{Modelling the Shape of the Earth}

$R$. Can you make the shape of the Earth with the green play-dough?

C. [Rolls ball then squeezes on desk with heels of hands to form flat rectangular shape].

R. Alright (interrogative)?

C. [Folds pancake inwards to form lump then rolls into ball again].

$R$. [I adjust modelling sheet to give child more room for modelling].

C. [Rolls ball on desk to form sphere]

R. That was a square shape you were making, was it?

$R$. [Earlier in interview child described the Earth as square and drew it as a rectangle.]

C. Yes-I'm trying to make it.

C. [Flattens ball to disc by squeezing and kneading with heel and palm of hand].

C. [Traced a square on disc shaped pancake as if cutting].

R. Are you wanting to cut it?

C. Yes. 


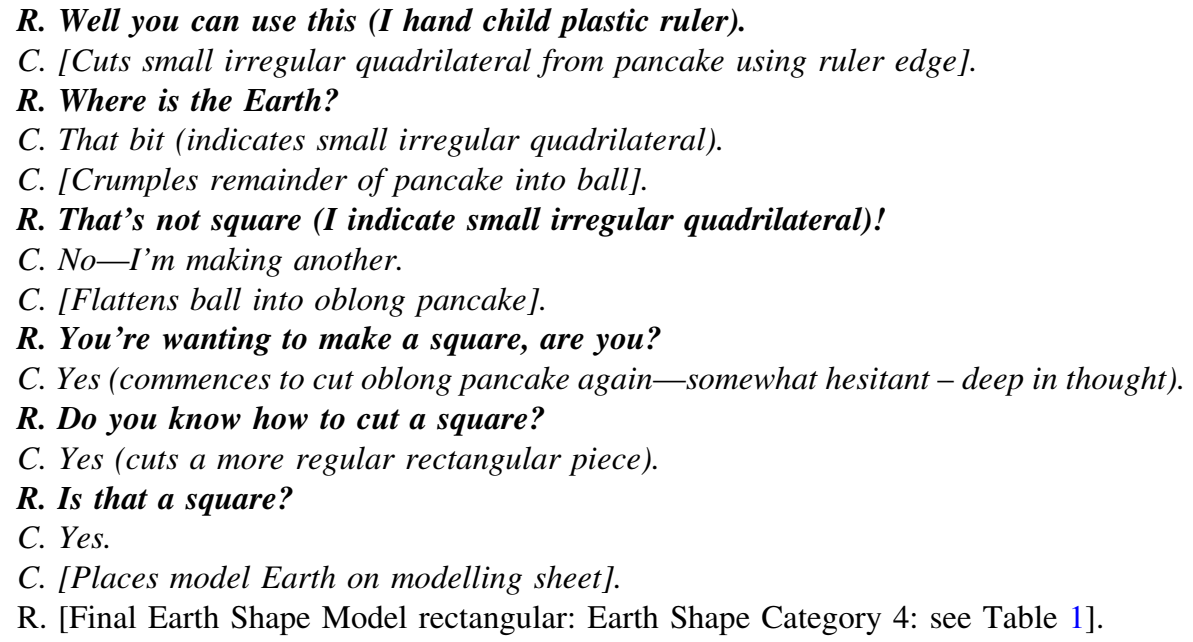

\section{Comparison between model and drawing}

R. Is that Earth (I indicate child's model) like that one (I indicate child's drawing)?

C. (Considers).

R. Is that (model) the same as that (drawing)?

C. No (attempts to re-orientate model which is placed horizontally so that it matches her drawing which shows a vertical rectangle).

R. No - don't move it - it's alright!

C. [Retracts hand from model].

$R$. Is that (drawing) like that (model)?

C. Yes.

\section{Modelling Ground and Sky}

$R$. If that's the Earth (I indicate child's model); where is the ground?

$R$. [I seek to ascertain whether the child holds the Earth and ground to be synonymous].

C. I'll make the ground.

R. No-just tell me.

C. [Considers].

$R$. Is the ground there or not?

C. No.

R. Where is the sky?

C. Up there (indicates area above models of Earth, Sun and Moon, as in her drawing).

$R$. [Sky is drawn as curve above Earth, Sun and Moon].

$\boldsymbol{R}$. Where would the ground be?

C. [Indicates area below model Earth].

R. Down there?

C. Yes.

R. [Linda drew the ground as square, below and separate from the Earth].

Initially, Linda made a rough ball shape which she flattened into a rough disc then further pressed into a thin pancake-like layer. She then folded the play-dough pancake and 
moulded it into a rough ball. This she shaped into a smooth ball that she pressed into a smooth disc and then a flat pancake from which she created an irregular shape similar to a quadrilateral using a ruler as a cutting tool. Dissatisfied with her initial shape, she remodelled the play-dough into a rough ball and repeated the process of flattening into a disc (pancake) from which she formed a second rectangular shape. Thus, the ball-shaped Earth afforded transformation into a disc shape by pressing, and the disc shape could be cut to produce a rectangular shape representing the everyday Earth. However, when asked to indicate the ground, Linda offered to make it, thus indicating that her model of the Earth did not incorporate the ground and was not synonymous with the ground. Interestingly, when comparing verbal descriptions with models and drawings, Linda attempted to reorientate her rectangular model of the Earth (with its major axis horizontal) to the same orientation as her drawing of the Earth (with its major axis vertical), suggesting that an unconscious matching process was taking place during modelling with the model being shaped to match the image of the drawing, and a conscious matching process taking place during later comparison guided by the researcher's questions. These comparisons between multiple modalities indicate dynamic conceptual simulations of coherent images of Earth, Sun and Moon Shape consistent with Barsalou's (2003) theory of concepts as simulators or skills.

The question arises as to whether Linda's intermediary ball-shaped and disc-shaped models represented the Earth or whether they were simply stages in the manipulation of the play-dough leading to a flat pancake from which a rectangular shape representing the Earth could be cut-much as a baker manipulates dough where the shape of the intermediate stages have no obvious relationship to the shape of the finished product. Based on observation of the modelling process alone, the question is difficult to answer. Fortunately, we have other media (verbal dialogue and drawing) to call upon, together with the knowledge of what the child has been taught in school to triangulate what the child may have had in mind. These indicate that Linda did indeed know of the scientific spherical Earth (she modelled the Sun and Moon as spherical) and she may well have considered this option but chose finally to model an earlier everyday concept of a flat Earth in the form of a square in preference to the spherical one.

\subsubsection{Takarangi (NZ: Māori: Male: Age 8 years 7 months: Junior Primary School)}

\section{Modelling the Shape of the Sun}

$R$. (Can you) make the shape of the Sun with the red play-dough?

$C$. [Creates ball-shaped model of the Sun by pressing and moulding between hands].

$\boldsymbol{R}$. What shape have you made there?

C. A little one like a pancake (unclear).

R. It's like a ball as well (as the Earth)?

$R$. [Child modelled Earth as ball-shaped].

C. It's probably like a pancake.

R. Like a pancake?

C. Yes.

R. If it's like a pancake, shouldn't it be flat (I gesture flatness with a pressing movement)?

C. Yes (flattens play-dough ball on desk top with right hand).

R. You think it's like a pancake?

C. Yes (continues to flatten ball to disc by pressing between hands).

R. You don't think it's like a ball? 
C. [No verbal response-continues to flatten shape to disc on knee].

R. Put it on here to flatten it (I indicate desk top).

C. OK (continues to flatten to disc on desk top by pressing with both hands).

R. [Final Sun Shape Model is a disc: Sun Shape Concept Category 4: see Table 1].

Takarangi initially modelled the Sun as a sphere but changed his mind when asked what shape he had made. He described his model as pancake-shaped, which conflicted with the physical model he had created which was ball-shaped. The interviewer's argument that a pancake is flat was immediately accepted by the child who then re-modelled his Sun as a flat disc. Although scientifically incorrect, the child was satisfied that his model of the Sun no longer conflicted with his mental image of the Sun which was consistent with his descriptions and drawings. The fact that his model Sun is disc-shaped, whereas his model Earth and model Moon are ball shaped does not strike the child as inconsistent at this stage of development. Takarangi tended to be preoccupied with creating his disc-shaped Sun in the modelling modality. Although prompted by Socratic dialogue to reconsider $[R$. You don't think it's like a ball?], he persevered with making his model Sun as he imagined it to be. His focus on the immediate kinesthetic modality is a "counter example" to the RQ. These protocols illustrate that children can hold both "confirming" and "disconfirming indicators" of the RQs during the same interview depending on the degree of interaction with the researcher through questions in the Interview Guide or through Socratic dialogue. As can be seen from the examples, the proportion of "confirming indicators" is much higher than that of "disconfirming indicators" adding further support to affirmative confirmation of the research questions.

\subsubsection{Shi Lin (China: Han: Female: Age 9 years 2 months: Senior Primary School)}

Modelling the Shape, Nature and Structure of the Moon with Play-dough

$R$. Make the shapes of the Moon with the yellow play-dough.

R. You made (drew) several Moon Shapes-four of them-so you can make all of those.

$R$. [I hand child four lumps of yellow play-dough].

$R$. [I introduce child's drawing of the Earth, Sun and Moon].

$R$. These are the Moon Shapes that you drew there (I indicate child's drawing).

C. (Makes first Moon Shape - a ball - by circular rolling between hands; then curves ball into cusp; then into disc by pressing between hands; then pressing on desk; then begins to tear apart but changes mind and squeezes back to ball; then refines ball shape by circular and horizontal rolling between hands and between hands and desk).

C. (Makes second Moon Shape - a crescent - by circular rolling between hands; then flattening ball to a disc by pressing on desk with right and left hand alternatively; then splitting disc into two semi-discs; and shaping one of the semi-discs into a crescent shape by gripping with right hand and tapping edge on palm of left hand as on an anvil; then tapping on desk; and moulding/forming with fingers).

R. You're making a good job of these.

C. (Makes third Moon Shape - a disc - by rolling into ball by circular rolling between hands; then pressing on desk with right hand to form disc).

R. It's a little thin one, the last one, isn't it?

C. (Makes fourth Moon Shape by switching back to discarded semi-disc from second Moon Shape, and shaping it into a crescent by pressing on desk with right hand, both 
hands, and left hand respectively; then trimming off the edges with her fingers using a tearing motion; and finally shaping and moulding edges with fingers).

C. (Returns to third Moon Shape - a disc -; flattens again by pressing on desk with both hands; then tears apart; rolls half between hands to form a ball; flattens ball to disc by pressing on desk; tears disc apart to form two semi-discs; and trims off edge by plucking off surplus play-dough).

$R$. [I comment that it is like making jiăozi-dumplings].

C. I know how to make dumplings.

$\boldsymbol{R}$. What shape is that Moon there (I indicate ball-shaped model Moon)?

C. Round like a ball.

$R$. Are all of these ones flat (I indicate the three crescent Moon Shapes)?

C. No-round like a pancake.

$\boldsymbol{R}$. Are these ones like parts of a pancake?

C. Yes.

R. [Model is spherical with phases: Moon Shape Category 10: see Table 1].

Shi Lin's modelling of Moon shapes demonstrates that, like most Chinese children of her age, she held coexisting cultural and scientific concepts of the Moon. This duality is manifest in Shi Lin's modelling the scientific Moon Shape as a ball and the crescentshaped phases of the Moon as semi-discs. The latter are visualised as sections of a pancake (bing) rather than sections of a ball or sphere or simply flat. Shi Lin's change of mind when creating her first Moon model-where she begins to tear her disc shape apart to create two semi-discs and then decides to revert to her original ball shape, may also reflect the tension between representing both the cultural and the scientific view. But it also shows that she had a clear idea of all of the shapes that she intended to make before she started modelling. This may have been influenced by cross-reference to her drawing but, in terms of showing the $3 \mathrm{D}$ aspects of the two main shapes, this seems unlikely. Throughout her modelling, Shi Lin appeared to have some form of mental reference to which she was referring shape by shape and hand motion by hand motion. Rather than some form of mental models or symbols in memory, it seemed more likely that she was creating comparisons from information in her mind by means of simulations (c.f. Barsalou 2003; Edelman 2005).

\subsection{Age, Gender, Culture and Ethnicity as Factors in Concept Creation}

The principal study of this research has focused on the qualitative analysis of the interview protocols. However, at the end of the section containing the research questions, we explained the need for a secondary, quantitative analysis checking for possible gender and culture/ethnicity effects on subjects' scores on the classification categories established for Earth Shape and motion (the categories shown in Tables 1, 2). As indicated earlier in relation to that analysis, we had hypothesised that:

H1 the dynamic structure of memory and concept creation will be similar in both genders and common across cultures [NZ boys $v$. NZ girls; China boys $v$. China girls; NZ v. China; NZ European v. NZ Māori];

H2 repeated interviews in this longitudinal study will lead to enhanced modelling skills and/or more advanced shape and motion concepts.

The results supported the hypotheses as shown in Tables 3 and 4. 
Table 3 Results of Kolmogorov-Smirnov two-sample test [K-S]: Modelling concept category

\begin{tabular}{|c|c|c|c|c|c|}
\hline Modelling concept & Culture & Gender & $N$ & $M$ & $p$ value \\
\hline \multicolumn{6}{|c|}{ (a) Testing Hypothesis H1: Gender: 2nd Survey: Boys versus Girls } \\
\hline \multirow[t]{4}{*}{ Earth Shape } & NZ & Boys & 34 & 8.82 & $p>.10$ \\
\hline & & Girls & 36 & 7.75 & \\
\hline & China & Boys & 32 & 8.75 & $p>.10$ \\
\hline & & Girls & 28 & 8.96 & \\
\hline \multirow[t]{4}{*}{ Earth Motion } & $\mathrm{NZ}$ & Boys & 34 & 6.41 & $p>.10$ \\
\hline & & Girls & 36 & 6.39 & \\
\hline & China & Boys & 32 & 6.53 & $p>.10$ \\
\hline & & Girls & 28 & 7.18 & \\
\hline
\end{tabular}

\begin{tabular}{lllll}
\hline Modelling concept & Culture & $N$ & $M$ & $p$ value \\
\hline
\end{tabular}

(b) Testing Hypothesis H1: Cross-Cultural: 1st NZ Survey versus 1st China Survey $[K-S]$

$\begin{array}{lllll}\text { Earth Shape } & \text { NZ } & 36 & 8.69 & p>.10 \\ \text { Earth Motion } & \text { China } & 36 & 8.50 & \\ & \text { NZ } & 36 & 6.00 & p>.10 \\ & \text { China } & 36 & 5.28 & \end{array}$

\begin{tabular}{lllll}
\hline Modelling concept & Ethnicity & $N$ & $M$ & $p$ value \\
\hline
\end{tabular}

(c) Testing hypothesis H1: Ethnicity: 2nd NZ Survey: NZ European versus NZ Māori [K-S]

\begin{tabular}{|c|c|c|c|c|}
\hline \multirow[t]{2}{*}{ Earth Shape } & NZ European & 22 & 8.41 & $p>.10$ \\
\hline & NZ Māori & 22 & 8.09 & \\
\hline \multirow[t]{2}{*}{ Earth Motion } & NZ European & 22 & 5.18 & $p>.10$ \\
\hline & NZ Māori & 22 & 4.77 & \\
\hline
\end{tabular}

There were no significant differences, but the means of NZ boys were greater than the means of NZ girls in both cases, whereas the means of China girls were greater than the means of China boys in both cases. A similar pattern was evident for the Sun and Moon. These results support H1 that the dynamic structure of memory and concept creation will be similar in both genders

Similar results were obtained from comparison of the 2nd Survey groups

The means of the NZ Survey group were higher than the China Survey group in all cases, but there were no significant differences. These findings support hypothesis $\mathrm{H} 1$ that the dynamic structure of memory and concept creation will be common across cultures

The NZ Māori participants were matched with a selection of the NZ European sample based on age, gender, general ability and socio-economic status (parent's occupations). There were no significant differences, but the means of NZ Europeans tended to be slightly greater than the means of NZ Māori. Results for the Sun and Moon were similar. The results are evidence in support of hypothesis H1 that the dynamic structure of memory and concept creation will be common across ethnicities (or ethnic groups)

\section{Conclusions}

\subsection{Support of Research Questions and Hypotheses}

The evidence here suggests that concept creation is a multifaceted dynamic process involving conceptual and motor skills and simulations as proposed by Barsalou (2003) and that these processes are compatible with Edelman's re-entry theory involving multiple 
Table 4 Testing Hypothesis H2: Results of Kolmogorov-Smirnov two-sample test [K-S]: Modelling concept category

\begin{tabular}{llllll}
\hline Modelling concept & Culture & Group & $N$ & $M$ & $p$ value \\
\hline Earth Shape & NZ & Survey & 36 & 8.58 & $p>.10$ \\
& & Control & 34 & 7.94 & \\
& China & Survey & 30 & 8.90 & $p>.10$ \\
& & Control & 30 & 8.77 & \\
Earth Motion & NZ & Survey & 36 & 6.58 & $p>.10$ \\
& & Control & 34 & 6.21 & \\
& China & Survey & 30 & 6.97 & $p>.10$ \\
& & Control & 30 & 6.70 & \\
\hline
\end{tabular}

Longitudinal: Survey group versus Control group

There were no significant differences between the Survey and Control groups in either culture $[K-S$ : $p>.10: \alpha .05]$. However, the means of the NZ \& China Survey groups were higher than the means of the Control groups in all cases. This suggests that repeated interviews in longitudinal studies of dexterity can result in Survey groups having more enhanced modelling skills and/or more advanced shape and motion concepts as a result of modelling experience. However, the impact of the repeated measures was not significant (as desired in longitudinal studies)

neural pathways and non-representative memory. Each child is unique and so is each child's cosmology. Although we can generalise, classify and categorise cosmological concepts to reveal overall patterns, at an individual level such schemes only approximate the end products of what a child believes; they say little about the process of concept creation. And because they are concerned with recording and reporting what they find as end products in a representational format, they tend to take for granted a representational view of memory.

However, from the perspective of the neural, psychological and physiological processes involved, a representational mode of presentation (and an underlying representational understanding of memory) appears to be inadequate and mismatched to the richness of the data available. It also fails to recognise the rapidity of conceptual change at an individual level, in terms of the multi-modal options being considered simultaneously. Through this study, we may not have sufficient evidence to completely reject mental models and representational memory, but we have shown that there is a more dynamic and flexible interpretation possible, one which recognises concepts as skills and simulations within an underlying theory of neural re-entry networks and non-representative memory. Having found that the RQs are supported in our samples suggests that they are likely to occur in the population in general.

The categorisation scheme which we used in this and previous research, and the methodology on which it is founded, has had the advantage over others ${ }^{6}$ in being multimodal and open-ended in nature: with inputs from interview, drawing and modelling triangulated to determine a category (of that cosmological element at that time). The scheme is more dynamic than those that rely on verbal language alone, or language with some drawing, or those based on forced-choice responses with limited opportunity for in-

\footnotetext{
${ }^{6}$ For example, see Nussbaum \& Novak (1976), Nussbaum (1979), Sneider and Pulos (1983), Vosniadou and Brewer (1992), all of which utilised verbal interviews with some opportunities for children's drawings but no play-dough (or equivalent) modelling by children themselves.
} 
depth probing of ideas through Socratic dialogue. The underlying procedure also limits the use of cultural artefacts likely to influence children's responses ${ }^{7}$ by avoiding pre-made models and globes. ${ }^{8}$ However, the difficulty for all researchers who utilise categorisation schemes is that children do not put into words everything that they think; nor do they draw every image that they imagine; nor do they create every shape that comes to mind when modelling. Researchers capture only a fraction of the concept creation process, and have to infer from that evidence the processes that are composing the action that they hear and see.

With the caveat expressed earlier in this article regarding the facilitating (and constraining) circumstances regarding semi-structured interviewing, the findings support all four research questions and both tentative hypotheses. The hand dexterity results (i.e. types of grips and hand motions used by children to shape and manipulate play-dough) are similar to those reported by others in the field (see Elliott and Connolly 1984; Napier 1956). Similarly, the Earth, Sun and Moon Shape and Motion results (i.e. Cosmological Concept Categories and Earth notions) are compatible with those that we and others have reported in the past (Bryce and Blown 2006, 2007, 2012, 2013; Blown and Bryce 2006, 2010, 2012; Nussbaum 1979; Nussbaum and Novak 1976; Sneider and Pulos 1983). However, the overall findings differ from those of other researchers in the field whose analyses have incorporated the term mental models with implications of representational memory (see Vosniadou and Brewer 1992, 1994). Our own results here support the alternative position that concepts are generated by cognitive and motor skills and simulations.

Methodologically, our results underline the necessity of probing beneath the surface appearances by Socratic dialogue in association with drawings and models, to reveal what children really think. Interviewing a child multi-modally is analogous to looking at a galaxy with different wavelengths of light. A galaxy may look quite uninteresting in visible light but spectacular in ultraviolet or infrared or sub-millimetre radiations. The research strategy we have adopted also points to an additional interpretation of Barsalou's view of concepts as skills: in an interview situation, the skill of the child is being honed by the skill of the interviewer. The experienced ethnographic researcher who is accepted, relaxed and not pressured by time will be able to obtain a much more accurate picture of a child's concept than will a stranger who is not used to children or familiar with the school community or wider culture, under time pressure, and using a methodology restricted to verbal language without Socratic dialogue.

\subsection{Levels of Analysis}

The study was limited to what can be known from analysis of children's verbal responses to Piagetian/Vygotskian interview questions utilising Socratic dialogue, children's drawings, and children's play-dough modelling sequences recorded with audio and video tape. The researchers did not have access to sophisticated hand motion analysis techniques involving computer modelling, capturing hand motion in image sequences (see Chien et al. 2010; Lin et al. 2002). However, we recognise that a more complete study allowing for greater qualitative and quantitative analysis could benefit from the application of such techniques. For example, we hypothesise that concept skill in the way that Barsalou uses

\footnotetext{
7 For example, see Frède et al. (2011), Nobes et al. (2003) and Panagiotaki et al. (2006), who found that changing the interview structure from open ended (based on Vosniadou and Brewer 1992) to forced choice, dramatically limited the range children's of Earth Shape categories.

8 See Schoultz et al. (2001), Vosniadou et al. (2005).
} 
the term involves an integration of cognitive skill and motor skill: the two being mutually intertwined and supportive (see Donaldson 1978) through neural re-entry and feedback circuits so that they develop in close unity (Edelman 2005).

But measurement of dexterous skill is limited by the ability of the human eye and brain to detect fine motor movement (as the researcher found when "freezing" individual motions at $1 / 25 \mathrm{~s}$ on video). As mentioned above, it was observed that some older children use apparently fewer gross hand motions than younger children to achieve the same result (e.g. to create a spherical-shaped model Earth) which confounded measurements based on recording gross motor motions alone. The reason being related to repeated handgrip motions (whereas a young child may take several different (gross) hand motions to create a spherical shape, an older child can complete the task in apparently fewer (gross) motions by utilising greater muscular strength and enhanced fine motor skills to mould the playdough by "pumping" with their hands). Such movements are difficult to detect without sophisticated apparatus. We believe that if these difficulties could be overcome the research questions might qualify some of the explanations, we have tendered. We can also see possible applications of brain imaging techniques such as MRI to record areas of activity as children model the shape and motion of the planetary objects, but such a programme on a large scale would be an expensive undertaking.

\subsection{The Coherence of Children's Conceptual Models}

In an earlier section, we discussed the coherence we have detected in children's conceptualisations in this field of developmental research, noting the correspondences between our own findings and those of Vosniadou et al. and the contrasts with those of diSessa's knowledge-in-pieces theory. The recent paper by Vosniadou and Skopeliti (2014) reconsiders the issues and makes a case for children's naïve physics (the understandings that children develop on the basis of their everyday experiences, observations and lay culture) being relatively coherent and not fragmented; that these understandings constitute a framework. Only with children's subsequent attempts to incorporate more formal (school) learning and often counter-intuitive concepts, does learning acquire fragmentation, the extent of it dependent, among other things, on how patient and sensitive is their school instruction: careful teachers can help children deal with cognitive conflict and prevent fragmentation taking place. The authors state that their framework theory accepts that children can hold internally inconsistent ideas: the "framework" imposes some general constraints upon their explanations but permits contextual variations and therefore conflicting details to exist and be apparent to teachers/researchers.

Vosniadou and Skopeliti's (2014) discussion is in line with our previous findings about conceptual coherence, and it is pertinent therefore to ask whether the findings we have provided in this paper shed any particular light on the coherence debate. We would argue that they do, that Edelman's stress upon the creative steps involved in the reproductive act of remembering can be developed further to understand what might be taking place during an interview. The data seems to suggest a creative or imaginative process in which composite coherent images are created from elements from a selection of alternative images with corresponding linguistic and modelling equivalents (analogous to concepts as skills and simulations). But these seem to be infinitely variable and contrary to mental models. For example, we found examples of images being rotated and reversed over time, such as images of the crescent Moon reversed by top-to-bottom rotation perpendicular to the page rather than by clockwise or anti-clockwise rotation between motion drawings and models a year apart, and switches in orientation of crescent Moons from facing right to 
facing left in consecutive drawings and models. These multi-modal variations in perspective are hard to explain by mental model theory but fit well with the idea that they are being created from a dynamic set of elements. Further evidence in support of Edelman's interpretation comes from instances where children transform spherical models to discshaped models and vice versa in a matter of seconds as they oscillate between 2D and 3D images (everyday versus scientific concepts) in their imagination.

\subsection{Concept Creation and Remembering}

Speculating further, when we try to recall what we know about something (that crops up in our observations/conversations/reactions to teacher or researcher questions, etc....) is the act of remembering a process where anything relevant-whether words, visual images, favourite sounds, artefacts of some sort-are brought to mind (not necessarily all instantly) and, at that point, an inventive process then takes place where a mental response is devised: hence the dynamism. Does the expression substrate hint at what we are working from creatively, but not deliberately or thoughtfully so at first? Does the created product then consciously constitute what we are remembering? Is Edelman correct to emphasise the creativeness but overdoing the condemnation of any and all mental representations? In his 2005 text, Edelman pressed the distinction between what is triggered in our minds when questioned being non-representational and the subsequent perceptual mappings being representational (thus affording conscious understanding, meaning or semantic content). "The essential issue to grasp is that the re-entrant circuitry underlying consciousness is enormously degenerate. There is no single circuit activity or code that corresponds to a given conscious 'representation'" (Edelman 2005, pp. 105-106).

Perhaps the expression used above-elements - points to what might be appropriate. If the creativeness involved in the recall process is correct, there should be

1. some similarities each time we are so questioned and respond (hence the coherence detectable),

2. increasing similarities each time we are questioned like this and respond accordingly,

3. some variations because what is available is changing with intervening learning and development, and

4. the creative act at any point might itself throw up different ideas depending on what is triggered first or what mindset is operating, ideas which, upon their articulation, could reconfigure what we are thinking of.

Also, as per the Dresp-Langley (2012) conclusions alluded to earlier, some unconscious thoughts of use may be triggered and alter the formulation we give to our response. The findings of our research fit well with this theorising, a particular case in point being the examples of images being rotated and reversed over time. These run contrary to reproduction and fit with creation (construction); something relevant is being used in the process, but something different and still relevant (or bettered) is produced (= remembered). As we interact with the world multi-modally, switching will be commonplace-though illustrations by their very nature are difficult though not impossible to capture, as this research has shown.

Points 1-4 above suggest that some Socratic questions may be better than others in influencing what a subject may bring to mind, thereby altering his/her creations. And of course being made to shift modes will make for differences. Future investigations might fruitfully consider the details which follow from particular Socratic questions. But, given that every interviewee is unique, the flow of questions and answers, and any alternatives, 
would be very difficult to track. At least future research should look at things in more detail than has been done in the past in order to try to separate the reproductive part from the creative parts of the remembering process. If there are elements, as described above, when does any repeated production constitute a mental model or, to use the traditional term, concept?

We opened this article with a quotation from literature, citing Mark Twain (1989, p. 421) as having said (no doubt with a touch of irony) that "You cannot depend on your eyes when your imagination is out of focus". This may be an apposite comment here, given its emphasis on the relationship between what we are thinking about or anticipating, what is in our "mind's eye" and what we look for and see. Perhaps we should recognise that at the heart of what we think of when trying to recollect something is a creative, or imaginative step in the process of memory which shapes what we choose to say (and see figuratively and possibly devise). Edelman's message would be that there is little or nothing programmed at this point; the brain's perceptual mappings permit a range of alternatives to be created and spelled out.

Other developments in cognitive scientific research corroborate this idea that mental models are constructed in a dynamic way at the point of recall and that imagery plays "a very large, even pivotal, role in memory" (Thomas 2014, p. 1). To cite a few of these developments:

- Gibbs (2005) argues that (image) schemas are not enduring mental representations but are temporary links between sensory experience and short-term, transitory conceptualizations of both concrete events and abstract ideas.

- Gauker (2011) considers that much of our thinking takes place via mental imagery without the help of concepts, though true conceptual thinking and the meaningfulness of ideas derives from language and how we communicate with each other-to which we would add — through multi-modal interactions: language (spoken, written, gesture), drawing and modelling.

- Monti and Osherson (2012) report that, almost counter-intuitively, the role of language in reasoning is confined to an initial stage where verbally presented information is encoded as non-verbal representations. These are subsequently manipulated by mental operations that are not based on the neural mechanisms which handle natural language.

Also our sensory-motor systems seem to play a rather different role in our thinking than has been hitherto thought:

- Gallese and Lakoff (2005) replace the original notion that knowledge consists of amodal, symbolic representations with the radical view that concepts are embodied, i.e. mapped within the sensory-motor system: it provides both the structure and the semantic content of knowledge. Consistent with Edelman's (2005) analysis of neural networks and how they work, this means that the sensory-motor system is multi-modal and not modular. And, as Gallese and Lakoff emphasise, language itself is inherently multimodal. Imagining grasping makes use of the same neural substrate as actually performing and perceiving grasping. Thus, imagining is a form of simulation-a mental simulation of an action or a perception, using much of the same neuronal circuitry as actually acting or perceiving.

This too squares with Barsalou's (2008) contentions.

And recent neurobiological, animal experimentation offers further corroborations: 
- The conventional view of memory consolidation has been that the hippocampus is responsible for the rapid encoding of ideas or events (working memory) and that the neocortex is involved in slow learning and the subsequent establishment of long-term memories. Tse's (2011) research shows that consolidation can occur rapidly if an appropriate schema exists into which new information can be integrated. Generalising from rats to humans, we can say that rapid memory formation is therefore dependent on previously learned schemas; when present, relevant schemas enable subjects to acquire new ideas quickly and effectively.

- Intriguingly, the finding of "mirror neurons" in monkeys by Rizzolatti and Craighero (1998) — whereby the same neuron is active when the animal either engages in an action, or observes another animal engaging in that same action-leads Nadel and Piattellli-Palmarini (2003) to write: "The existence of such neurons raises questions of great import to philosophers of mind... The possible role of a system of mirror neurons in the creation of internal mental models is obvious" (p. 32).

Clearly, the research described in this article has concentrated, in terms of data, upon children's ideas about observational astronomy and it will be for future researchers to explore other areas of knowledge, seek corroboration and further clarifications to how we should understand the processes concerned. The problem of how people reason is important because the nature of concepts and the structure of human memory are fundamental components of psychology. If concepts are indeed dynamic skills and simulations, and if memory is indeed non-representational, then the implications for developmental research will be quite revolutionary. With regard to teaching, it is clear that not every individual learner will naturally activate cross-modality checks upon their thinking. This suggests that in learning environments greater attention might be paid by teachers to encourage such checks during the course of their practice.

Conflict of interest The authors declare that they have no conflict of interest.

Open Access This article is distributed under the terms of the Creative Commons Attribution 4.0 International License (http://creativecommons.org/licenses/by/4.0/), which permits unrestricted use, distribution, and reproduction in any medium, provided you give appropriate credit to the original author(s) and the source, provide a link to the Creative Commons license, and indicate if changes were made.

\section{References}

Adúriz-Bravo, A. (2013). A 'semantic' view of scientific models for science education. Science \& Education, 22(7), 1593-1611.

Barsalou, L. W. (2003). Situated simulation in the human conceptual system. Language and Cognitive Processes, 18(5/6), 513-562.

Barsalou, L. W. (2008). Grounded Cognition. Annual Review of Psychology, 59, 617-645.

Blown, E. J., \& Bryce, T. G. K. (2006). Knowledge restructuring in the development of children's cosmologies. International Journal of Science Education, 28(12), 1411-1462.

Blown, E. J., \& Bryce, T. G. K. (2010). Conceptual coherence revealed in multi-modal representations of astronomy knowledge. International Journal of Science Education, 32(1), 31-67.

Blown, E. J., \& Bryce, T. G. K. (2012). Thought-experiments about gravity in the history of science and in research into children's thinking. Science \& Education, 22(3), 419-483.

Brewer, W. F., Herdrich, D. J., \& Vosniadou, S. (1987). A cross-cultural study of children's development of cosmological models: Samoan and American data. Paper presented at the Third International Conference on Thinking, Honolulu, HI.

Bryce, T. G. K., \& Blown, E. J. (2006). Cultural mediation of children's cosmologies: A longitudinal study of the astronomy concepts of Chinese and New Zealand children. International Journal of Science Education, 28(10), 1113-1160. 
Bryce, T. G. K., \& Blown, E. J. (2007). Gender effects in children's development and education. International Journal of Science Education, 29(13), 1655-1678.

Bryce, T. G. K., \& Blown, E. J. (2012). The novice-expert continuum in astronomy knowledge. International Journal of Science Education, 34(4), 545-587.

Bryce, T. G. K., \& Blown, E. J. (2013). Children's concepts of the shape and size of the Earth, Sun and Moon. International Journal of Science Education, 35(3), 388-446.

Chien, C.-W., Brown, T., \& McDonald, R. (2010). Examining content validity and reliability of children's hand skills (ACHS): A preliminary study. American Journal of Occupational Therapy, 64(5), 756-767.

Clancey, W. J. (1991). Review of Rosenfield's “The invention of memory”. Artificial Intelligence, 50(2), 241-284.

Clancey, W. J. (1999). Conceptual coordination: How the mind orders experience in time. Mahwah: Lawrence Erlbaum.

de Hosson, C., \& Décamp, N. (2014). Using ancient Chinese and Greek astronomical data: A training sequence in elementary astronomy for pre-service primary school teachers. Science \& Education, 23(4), 809-827.

diSessa, A. A. (1988). Knowledge in pieces. In G. Forman \& P. B. Pufall (Eds.), Constructivism in the computer age (pp. 49-70). Mahwah: Lawrence Erlbaum.

diSessa, A. A. (2008). A bird's eye view of the "pieces" versus "coherence" controversy from the "pieces" side of the fence. In S. Vosniadou (Ed.), International handbook of research on conceptual change (pp. 35-60). London: Routledge.

Donaldson, M. (1978). Children's minds. Glasgow: William Collins and Sons.

Dresp-Langley, B. (2012). Why the Brain knows more than we do: Non-conscious representations and their role in the construction of conscious experience. Brain Sciences, 2, 1-21.

Dreyfus, A., Jungwirth, E., \& Eliovitch, R. (1990). Applying the "cognitive conflict" strategy for conceptual change-some implications, difficulties, and problems. Science Education, 74(5), 555-569.

Ebison, M. G. (1993). Newtonian in mind but Aristotelian at heart. Science \& Education, 2(4), $345-362$.

Edelman, G. M. (2005). Wider than the Sky: A revolutionary view of consciousness. London: Penguin.

Edelman, G. M. (2006). Second nature: Brain science and human knowledge. New Haven: Yale University Press.

Edelman, G. M., \& Tononi, G. (1996). Commentary-Selection and development: the brain as a complex system. In D. Magnusson (Ed.), Chapter 9 in the lifespan development of individuals behavioral, neurobiological and psychosocial perspectives. Cambridge: Cambridge University Press.

Elliott, J. M., \& Connolly, K. J. (1984). A classification of manipulative hand movements. Developmental Medicine and Child Neurology, 26, 283-296.

Feigenberg, J., Larvik, L. V., \& Shunyakov, V. (2002). Space scale: Models in the history of science and students mental models. Science \& Education, 11(4), 377-392.

Frède, V., Nobes, G., Frappart, S., Panagiotaki, G., Troadec, B., \& Martin, A. (2011). The acquisition of scientific knowledge. The influence of methods of questioning and analysis on the interpretation of children's conceptions of the Earth. Infant and Child Development, 20, 432-448.

Galili, I. (2009). Thought experiments: Determining their meaning. Science \& Education, 18(1), 1-23.

Gallagher, S. (2005). How the body shapes the mind. Oxford: Oxford University Press.

Gallese, V., \& Lakoff, G. (2005). The Brain's Concepts: The role of the sensory-motor system in conceptual knowledge. Cognitive Neuropsychology, 22(3/4), 455-479.

Gauker, C. (2011). Words and images: An essay on the origin of ideas. Oxford: Oxford University Press.

Gentner, D., \& Stevens, A. L. (Eds.). (1983). Mental models. New York: Psychology Press.

Gibbs, R. W. (2005). The psychological status of image schemas. In B. Hampe \& J. E. Grady (Eds.), From perception to meaning: image schemas in cognitive linguistics. Berlin: Walter de Gruyter BmbH \& Co.

Gilbert, J. K., \& Boulter, C. (Eds.). (2012). Developing models in science education. London: Kluwer Academic.

Grandy, R. E. (2003). What are models and why do we need them? Science \& Education, 12(8), $773-777$.

Halloun, I. A. (2006). Mediated modelling in science education. Science \& Education, 16(7), 653-697.

Johnson-Laird, P. N. (1983). Mental models: Towards a cognitive science of language, inference and consciousness. Massachusetts: Harvard University Press.

Justi, R. S., \& Gilbert, J. K. (2002). Modelling, teachers' views on the nature of modelling, and implications for the education of modellers. International Journal of Science Education, 24(4), 369-387.

Klein, C. A. (1982). Children's concepts of the Earth and the Sun: A cross cultural study. Science Education, 65(1), 95-107.

Kösem, S. D., \& Özdemir, Ö. F. (2014). The nature and role of thought experiments in solving conceptual physics problems. Science \& Education, 23(4), 865-895.

Lauginie, P. (2013). How did light acquire a velocity? Science \& Education, 22(6), 1537-1554. 
Lin, J., Wu, Y. \& Huang, T. S. (2002). Capturing human hand motion in image sequences. Motion and Video Computing Proceedings of Workshop. doi:10.1109/MOTION.2002.1182220

Martin, A. (2001). Functional neuroimaging of semantic memory. In R. Cabeza \& A. Kingstone (Eds.), Handbook of functional neuroimaging of cognition (pp. 153-186). Cambridge: MIT Press.

Martin, A. (2007). The representation of object concepts in the brain. Annual Review of Psychology, 58, $25-45$.

Matthews, M. R. (2007). Models in science and in science education: An introduction. Science \& Education, 16(7), 647-652.

McNeill, D. (1992). Hand and mind: what gestures reveal about thought. Chicago: The University of Chicago Press.

Monti, M. M., \& Osherson, D. N. (2012). Logic Language and the Brain. Brain Research, 1428(5), 33-42.

Murphy, G. L., \& Medin, D. L. (1985). The role of theories in conceptual coherence. Psychological Review, 92(3), 289-316.

Nadel, L., \& Piattelli-Palmarini, M. (2003). What is cognitive science? Encyclopedia of cognitive science (pp. 13-15). Hoboken: Wiley.

Napier, R. (1956). The prehensile movements of the human hand. Journal of Bone and Joint Surgery, British, 38(4), 902-913.

Nobes, G., Moore, D. G., Martin, A. S., Clifford, B. R., Butterworth, G., Panagiotaki, G., \& Siegal, M. (2003). Children's understanding of the Earth in a multicultural community: Mental models or fragments of knowledge? Developmental Science, 6, 72-85.

Nussbaum, J. (1979). Children's conceptions of the earth as a cosmic body: A cross age study. Science Education, 63, 83-93.

Nussbaum, J., \& Novak, J. D. (1976). An assessment of children's concepts of the earth utilizing structured interviews. Science Education, 60, 535-550.

Panagiotaki, G., Nobes, G., \& Banerjee, R. (2006). Is the world round or flat? Children's understanding of the Earth. European Journal of Developmental Psychology, 3, 124-141.

Piaget, J. (1929). The child's conception of the world. London: Routledge \& Kegan Paul.

Piaget, J. (1930). The child's conception of physical causality. London: Routledge \& Kegan Paul.

Piaget, J. (1970). Genetic epistemology. New York: W. W. Norton and Company.

Piaget, J. (1985). The equilibration of cognitive structures. Chicago: University of Chicago Press.

Reiner, M., \& Burko, L. M. (2003). On the limitations of thought experiments in physics and the consequences for physics education. Science \& Education, 12(4), 365-385.

Rizzolatti, G., \& Craighero, L. (1998). Spatial attention: Mechanisms and theories. In M. Sabourin, F. Craik, \& M. Robert (Eds.), Advances in psychological science, Volume 2: Biological and cognitive aspects. Hove: Psychology Press.

Rose, S. (2006). The 21st Century Brain: Explaining, mending and manipulating. London: Jonathan Cape.

Rose, S. (2007). Thinking allowed: Review of Second Nature: Brain Science and Human Knowledge by Gerald M. Edelman. The Guardian, Saturday 17 February.

Rosenfield, I. (1988). The invention of memory: A new view of the Brain. New York: Basic Books.

Schnotz, W., \& Preuß, A. (1997). Task-dependent construction of mental models as a basis for conceptual change. European Journal of Psychology of Education, 12(2), 185-211.

Schoultz, J., Säljö, R., \& Wyndhamn, J. (2001). Heavenly talk: Discourse, artifacts, and children's understanding of elementary astronomy. Human Development, 44, 103-118.

Sneider, C., \& Pulos, S. (1983). Children's cosmographies: Understanding the earth's shape and gravity. Science Education, 67, 205-221.

Spiliotopoulou-Papantoniou, V. (2006). Models of the universe: Children's experiences and evidence from the history of science. Science \& Education, 16(7), 801-833.

Thijs, G. D., \& Van Den Berg, E. (1995). Cultural factors in the origin and remediation of alternative conceptions in physics. Science \& Education, 4(4), 317-347.

Thomas, N. J. T. (2014). Imagery. Entry in Stanford Encyclopedia of Philosophy. http://plato.stanford.edu/ entries/mental-imagery/

Tse, D. (2011). Schema and Memory Consolidation. Doctoral Thesis, University of Edinburgh.

Twain, M. (1989). A Connecticut Yankee in King Arthur's Court. New York: Harper \& Brothers Publishers.

Van Gelder, T. (1998). The dynamical hypothesis in cognitive science. Behavioral and Brain Sciences, 21(5), 615-628.

Vosniadou, S. (1992). Knowledge acquisition and conceptual change. Applied Psychology, an International Review, 41(4), 347-357.

Vosniadou, S., \& Brewer, W. F. (1992). Mental models of the Earth: a study of conceptual change in childhood. Cognitive Psychology, 24(4), 535-585. 
Vosniadou, S., \& Brewer, W. F. (1994). Mental models of the day/night cycle. Cognitive Science, 18(1), $123-183$.

Vosniadou, S., \& Skopeliti, I. (2014). Conceptual change from the framework theory side of the fence. Science \& Education, 23, 1427-1445.

Vosniadou, S., Skopeliti, I., \& Ikospentaki, K. (2005). Reconsidering the role of artifacts in reasoning: Children's understanding of the globe as a model of the earth. Learning and Instruction, 15, 333-351.

Vosniadou, S., Vamvakoussi, X., \& Skopeliti, I. (2008). The framework theory approach to the problem of conceptual change. In S. Vosniadou (Ed.), International handbook of research on conceptual change (pp. 35-60). New York: Routledge.

Vygotsky, L. (2012). Thought and language (A. Kozulin Ed. \& Trans.) Cambridge, MIT Press (Original work published 1934) 